\title{
THE DISTRIBUTION OF ACTIVE GALACTIC NUCLEI IN CLUSTERS OF GALAXIES
}

\author{
Paul Martini \\ AND \\ John S. Mulchaey and Daniel D. Kelson \\ Carnegie Observatories, Pasadena, CA 91101-1292 \\ Received 2007 January 20; accepted 2007 April 18
}

Department of Astronomy, Ohio State University, Columbus, OH 43210; martini@astronomy.ohio-state.edu

\begin{abstract}
We present a study of the distribution of AGNs in clusters of galaxies with a uniformly selected, spectroscopically complete sample of 35 AGNs in eight clusters of galaxies at $z=0.06 \rightarrow 0.31$. We find that the 12 AGNs with $L_{\mathrm{X}}>10^{42} \mathrm{ergs} \mathrm{s}^{-1}$ in cluster members more luminous than a rest-frame $M_{R}<-20$ mag are more centrally concentrated than typical cluster galaxies of this luminosity, although these AGNs have comparable velocity and substructure distributions to other cluster members. In contrast, a larger sample of 30 cluster AGNs with $L_{\mathrm{X}}>10^{41} \mathrm{ergs} \mathrm{s}^{-1}$ do not show evidence for greater central concentration than inactive cluster members, nor evidence for a different kinematic or substructure distribution. As we do see clear differences in the spatial and kinematic distributions of the blue Butcher-Oemler and red cluster galaxy populations, any difference in the AGN and inactive galaxy population must be less distinct than that between these two pairs of populations. Comparison of the AGN fraction selected via $\mathrm{X}$-ray emission in this study to similarly selected AGNs in the field indicates that the AGN fraction is not significantly lower in clusters, contrary to AGNs identified via visible wavelength emission lines, but similar to the approximately constant radio-selected AGN fraction in clusters and the field. We also find significant evidence for variation in the AGN fraction between clusters and explore the dependence of cluster AGN fraction on redshift, velocity dispersion, amount of cluster substructure, and fraction of Butcher-Oemler galaxies. While we see weak evidence for several trends, there are several correlations between these four parameters in our small sample of eight clusters that preclude identification of which one(s) most strongly influence the cluster AGN fraction.
\end{abstract}

Subject headings: galaxies: active — galaxies: clusters: general — galaxies: evolution $-\mathrm{X}$-rays: galaxies $\mathrm{X}$-rays: galaxies: clusters $-\mathrm{X}$-rays: general

Online material: color figures, machine-readable table

\section{INTRODUCTION}

Early work on clusters of galaxies found that emission-line galaxies and active galactic nuclei (AGNs) were rarer in cluster members than in regions of lower galaxy density (Osterbrock 1960; Gisler 1978; Dressler et al. 1985). Extensive work on cluster galaxy populations demonstrated that cluster galaxies were dominated by early-type galaxies with old stellar populations and the fraction of these quiescent galaxies increased with galaxy surface density (Dressler 1980). These differences are now ascribed to multiple physical mechanisms that cause cluster members to possess less of the cold gas necessary for young star formation and accretion onto supermassive black holes.

The relative rarity of AGNs in clusters of galaxies made it difficult to acquire sufficiently large samples for demographic studies. For example, Dressler et al. (1985) obtained spectra of over 1000 cluster members in 10 low-redshift clusters of galaxies and identified AGNs in only $1 \%$ of them. This low fraction discouraged large spectroscopic studies whose primary purpose was to identify AGNs in clusters of galaxies. However, the advent of the Chandra X-Ray Observatory has provided a new means to efficiently identify AGNs in clusters. Motivated by early evidence of an excess of X-ray point sources in the fields of rich clusters of galaxies (Cappi et al. 2001; Sun \& Murray 2002; Molnar et al. 2002), Martini et al. (2002) obtained spectra of the bright counterparts to X-ray sources in the field of the rich cluster Abell $2104(z=0.154)$. This study identified six bright cluster galaxies coincident with luminous X-ray sources $\left(L_{\mathrm{X}}>\right.$ $10^{41} \mathrm{ergs} \mathrm{s}^{-1}$ ), or approximately $5 \%$ of cluster members more luminous than $M_{R}=-20 \mathrm{mag}$. While only the most X-ray luminous of these six galaxies have the emission-line diagnostics of AGNs, the remaining X-ray sources are more likely powered by accretion onto a supermassive black hole than other plausible sources of lower luminosity X-ray emission, such as a population of low-mass X-ray binaries (LMXBs), hot halos of diffuse gas, or star formation.

Motivated by the high AGN fraction in Abell 2104, we extended our survey to seven additional, low-redshift clusters of galaxies $(z=0.06 \rightarrow 0.31)$ and found that approximately $5 \%$ of galaxies more luminous than $M_{R}=-20$ mag host AGNs more luminous than $L_{\mathrm{X}}=10^{41} \mathrm{ergs} \mathrm{s}^{-1}$ in the broad $(0.5-8 \mathrm{keV})$ $\mathrm{X}$-ray band (Martini et al. 2006, hereafter Paper I). As was the case for Abell 2104, we found that most of the cluster galaxies with X-ray counterparts did not show obvious AGN spectral signatures in visible wavelength spectra. As LMXBs or thermal emission from hot halos have also been observed to produce luminous X-ray emission, particularly from bright, early-type galaxies, we used the multiwavelength spectral shape of these sources to determine if they were AGNs. Comparison with relations between X-ray and $B$-band luminosity for local early-type galaxies dominated by LMXBs (Kim \& Fabbiano 2004) and hot gas (O'Sullivan et al. 2003; Sun et al. 2005) showed that these AGN candidates were on order 1-3 orders of magnitude more X-ray luminous than expected from relations based on these other 
sources of X-ray emission. We therefore concluded that most of these sources were AGNs. Only a small fraction remain consistent with other sources of X-ray emission, and they are not included in the present study.

The goal of seeking a larger sample of AGNs in clusters of galaxies was to use the AGN population to explore the mechanisms responsible for fueling AGNs, motivated by the similar use of the cluster environment to explore other aspects of galaxy evolution. Comprehensive studies of clusters of galaxies have shown that the cluster environment contains a profoundly different distribution of galaxy populations from the field. The morphology-density relation expresses the observation that lenticular and then elliptical galaxies are dominant in progressively richer galaxy environments (Dressler 1980). The amount of current star formation in galaxies also declines toward the center of clusters (Fisher et al. 1998). Galaxies at the centers of rich clusters tend to be dominated by old stellar populations and have no active star formation, while a progressively higher fraction of poststarburst and starburst galaxies are found toward the outskirts.

Kinematic studies of cluster galaxies show that the population of galaxies with current or recent star formation have the highest velocity dispersions, poststarburst galaxies are intermediate, while the galaxy population dominated by a passive stellar population has the lowest velocity dispersion (Dressler et al. 1999). The higher velocity dispersions (and greater radial extent) suggest that galaxies with current or recent star formation are less virialized than more passive galaxies. These galaxies with young stellar populations may on average have entered the cluster more recently and have higher line-of-sight velocity dispersions because they remain on primarily radial orbits.

The distribution of AGNs in clusters of galaxies could provide similar information on the origin of AGNs in clusters. In particular, their distribution is a valuable test of the standard paradigm for AGN fueling, namely, the merger of two gas-rich galaxies (e.g., Barnes \& Hernquist 1992). The low AGN fraction in clusters is commonly ascribed to the lower merger rate in clusters due to the high velocity dispersion that precludes the formation of bound pairs, in spite of the high galaxy density and the lower fraction of galaxies with substantial reservoirs of cold gas (e.g., Giovanelli \& Haynes 1985). If this picture is correct, then AGNs should be more common at the outskirts of clusters where members are relatively rich in cold gas, as well as in lower velocity dispersion clusters. Many galaxies also enter the cluster potential in low velocity dispersion groups that may produce a relative increase in the AGN fraction at larger distances from the cluster center or associated with distinct substructure within the clusters. These relatively recent entrants into the cluster potential would also have not yet virialized and could have a larger velocity dispersion than the old, passively evolving galaxies at the center of the cluster potential.

In studies of lower velocity dispersion groups, Shen et al. (2007) found AGNs in $\sim 7 \%$ of galaxies at $z \sim 0.06$, yet these AGNs were only identified in visible wavelength spectroscopy and were not detected in their X-ray (XMM-Newton) observations and must have $L_{\mathrm{X}}<10^{41} \mathrm{ergs} \mathrm{s}^{-1}$. This result suggests that the X-ray and visible wavelength properties of typical AGNs in lower density environments may be different from the typical AGNs in higher density environments. Previously, Best et al. (2005) showed that the fraction of AGNs selected by emission lines from SDSS slightly decreases for galaxies with a larger number of luminous neighbors, yet the fraction of radio-selected AGNs actually increases in galaxies in richer environments. This is comparable to results from the field by Lehmer et al. (2007), who studied X-ray emission from early-type galaxies in the Ex- tended Chandra Deep Field-South and found an average AGN fraction with X-ray luminosity above $10^{41} \mathrm{ergs} \mathrm{s}^{-1}$ that is consistent with our measurement in clusters. For the broad X-ray band they find $f_{A}\left(M_{R}<-20 ; L_{\mathrm{X}}>10^{41}\right) \sim 7 \%$ and $f_{A}\left(M_{R}<-20\right.$; $\left.L_{\mathrm{X}}>10^{42}\right)=2 \%$ (B. Lehmer 2006, private communication). These fractions are remarkably similar to the mean AGN fractions we measure in clusters and may suggest that the X-rayluminous AGN fraction is not a strong function of environment, similar to the result of Best et al. (2005) for radio-luminous AGNs, although this does not explain the absence of X-rayluminous AGNs in the group study of Shen et al. (2007).

AGNs in clusters of galaxies have also garnered significant recent interest as an explanation of the absence of substantial cold gas at the centers of many clusters. Simple radiative cooling models predict that the intracluster medium (ICM) in the cores of many clusters should cool in less than a Hubble time, yet the predicted substantial reservoirs of cold gas are not observed. The presence of powerful radio galaxies at the centers of most predicted "cool core" clusters (e.g., Burns 1990) suggests that while the cooling gas may provide fuel for the AGNs, the AGNs may also be reheating the cool gas. The substantial cavities in the hot ICM coincident with the lobes of these radio galaxies demonstrate that the AGNs inject copious amounts of energy into the ICM (McNamara et al. 2000; Fabian et al. 2000). Bîrzan et al. (2004) have shown that the energy necessary to reheat the ICM and prevent cooling is approximately consistent with the amount of energy necessary to create the large cavities observed in the ICM, although the mechanism by which the highly collimated AGN jets uniformly heat the core ICM is still under active investigation. One possible resolution to the problem of uniform heating is multiple AGNs in the cluster core $(\sim 100 \mathrm{kpc})$. Nusser et al. (2006) recently showed that gas cooling at the centers of clusters could condense onto any galaxies within the core, fuel accretion onto the supermassive black holes, and produce more distributed heating of the ICM. A simple test of this scenario is to search for multiple or off-center AGNs in the cores of clusters. It is also important to quantify the population and evolution of radio-bright AGNs in the cores of clusters for future experiments that employ the Sunyaev-Zel'dovich effect to identify clusters because these sources could be a significant contaminant (e.g., Coble et al. 2006).

In the present paper we employ the other cluster members identified in our multiwavelength survey to derive the spatial and kinematic distribution of the cluster AGNs relative to the cluster galaxy population. We also use these data to derive velocity dispersions and study the amount of star formation present in these clusters to determine if any global cluster properties correlate with the cluster AGN fraction. In the next section we provide a brief summary of the observations described in Paper I, followed by a derivation of the velocity dispersion, membership, and visible wavelength properties of the cluster galaxies in $\S 3$. The distribution of the AGNs relative to other cluster members is described in $\S 4$, and the relation between AGN fraction and the properties of the cluster is discussed in $\S 5$. We present our conclusions in $\S 6$. Throughout this paper we assume that the cosmological parameters are $\left(\Omega_{M}, \Omega_{\Lambda}, h\right)=(0.3,0.7,0.7)$, where $H_{0}=100 h \mathrm{~km} \mathrm{~s}^{-1} \mathrm{Mpc}^{-1}$.

\section{OBSERVATIONS}

We identified AGNs in clusters of galaxies with X-ray and visible wavelength images and spectroscopy of the eight clusters of galaxies listed in Table 1. As AGNs are quite luminous at X-ray wavelengths, AGN candidates were identified as galaxies with X-ray counterparts. These galaxies were then observed 
TABLE 1

Cluster Properties

\begin{tabular}{|c|c|c|c|c|c|c|c|}
\hline $\begin{array}{l}\text { Cluster } \\
\text { (1) }\end{array}$ & $\begin{array}{l}\alpha_{c} \\
\text { (2) }\end{array}$ & $\begin{array}{c}\delta_{c} \\
(3)\end{array}$ & $\begin{array}{c}z \\
(4)\end{array}$ & $\begin{array}{c}z_{1}, z_{2} \\
(5)\end{array}$ & $\begin{array}{c}\sigma \\
(6)\end{array}$ & $\begin{array}{c}r_{200} \\
(\mathrm{Mpc}) \\
(7)\end{array}$ & $\begin{array}{l}P_{\delta} \\
(8)\end{array}$ \\
\hline 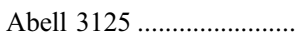 & 032717.9 & -532937 & 0.0616 & $0.0530,0.0700$ & $475(94)$ & 1.14 & $<0.001$ \\
\hline Abell 3128 & 033043.8 & -523130 & 0.0595 & $0.0435,0.0755$ & $906(74)$ & 2.18 & $<0.001$ \\
\hline 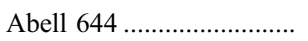 & 081725.6 & -073045 & 0.0701 & $0.0531,0.0871$ & $952(382)$ & 2.28 & 0.236 \\
\hline Abell 2104 & 154007.9 & -031816 & 0.1544 & $0.1304,0.1783$ & $1242(194)$ & 2.85 & 0.203 \\
\hline Abell 1689 & 131129.5 & -012028 & 0.1867 & $0.1392,0.2343$ & $2402(357)$ & 5.41 & 0.994 \\
\hline Abell 2163 ............................ & 161549.0 & -060841 & 0.2007 & $0.1731,0.2236$ & $1381(324)$ & 3.09 & 0.283 \\
\hline MS 1008 & 101032.4 & -123953 & 0.3068 & $0.2921,0.3215$ & $1127(153)$ & 2.38 & 0.722 \\
\hline AC 114 & 225848.4 & -344808 & 0.3148 & $0.2884,0.3412$ & 2025 (217) & 4.25 & 0.156 \\
\hline
\end{tabular}

Notes.- Units of right ascension are hours, minutes, and seconds, and units of declination are degrees, arcminutes, and arcseconds. Cluster sample and properties derived from the present study. Col. (1): Cluster name. Cols. (2) and (3): Right ascension and declination of the cluster center for epoch J2000.0. Col. (4): Redshift. Col. (5): Redshift range of cluster members. Col. (6): Velocity dispersion and uncertainty. Col. (7): Physical radius $r_{200}$. Col. (8): Probability that the observed substructure is consistent with a random redistribution of the cluster radial velocities. The derivation of these quantities is discussed in $\S \S 3$ and 4 .

spectroscopically to determine if they were members of a given cluster. This X-ray selection technique was employed for two reasons. First, AGNs are relatively rare in clusters of galaxies, and it is more efficient to obtain complete spectroscopic observations of all AGN candidates than to spectroscopically observe all bright galaxies because the surface density of candidate cluster members with X-ray counterparts is substantially lower than the surface density of all candidate cluster members. Second, $\mathrm{X}$-ray selection is a relatively unbiased method of identifying AGNs over a wide range of redshifts. Most AGNs are low luminosity (e.g., $L_{\mathrm{X}}<10^{43} \mathrm{ergs} \mathrm{s}^{-1}$ ), and these AGNs are difficult to identify spectroscopically at even low redshift (e.g., $z \sim 0.1$ ) due to dilution by host galaxy starlight and/or obscuration. X-ray emission from an AGN has substantially higher contrast over other sources of X-ray emission to much lower accretion power, particularly when the spectroscopic aperture includes a substantial fraction of the total emission of the galaxy. X-ray emission is also much less affected by obscuration than visible wavelength emission.

The observing strategy was therefore to compare visible wavelength and X-ray images of each cluster to identify potential cluster AGNs for follow-up spectroscopy. As discussed in greater detail in Paper I, the X-ray observations are archival Chandra observations of low-redshift clusters with sufficient sensitivity to identify low-luminosity AGNs. We obtained visible wavelength observations of the clusters with the $2.5 \mathrm{~m}$ du Pont Telescope at Las Campanas Observatory with either the WFCCD or Tek5 CCD cameras. $R$-band images of the centers of six of these clusters are shown in Figure 1, along with X-ray and radio contours. Comparable figures for Abell 3125 and Abell 3128 are presented in Rose et al. (2002). All X-ray counterparts brighter than $R \sim 23$ mag were then selected for multislit spectroscopic observations. As the surface density of X-ray counterparts was substantially less than the potential packing density of spectroscopic slits on a given mask, additional bright galaxies were observed to determine the spectroscopic properties of cluster members without X-ray emission, measure the fraction of cluster members with X-ray emission, and in several cases derive the velocity dispersion of the cluster for the first time.

Table 2 presents a catalog of all of our successful spectroscopic observations. As noted above, these targets were selected through a variety of algorithms. First, the highest priority was assigned to all X-ray sources brighter than $R \sim 23$ mag. Additional candidate cluster members were then targeted for spec- troscopy based on brightness, color, and availability of a spectroscopic slit. We successfully measured spectroscopic redshifts for all sources brighter than a rest-frame absolute magnitude of $M_{R}=-20 \mathrm{mag}$ at each cluster redshift. For the nearby clusters, the apparent magnitude limits extend substantially fainter. In general, we are complete to $R=21 \mathrm{mag}$ for all of these fields and to $R=22 \mathrm{mag}$ for all sources selected as X-ray counterparts. In addition, there are many fainter sources with emission-line redshifts. The exception is the Abell 2163 field, where the 90\% completeness for spectroscopy is approximately $R=20.5 \mathrm{mag}$ for both selection criteria. With the exception of Abell 644 and Abell 2163, there are literature redshift data for all of these fields. We observed a number of these sources in each field and used these data to determine that the typical redshift uncertainty of our observations is less than $\sigma_{z}=0.0005$.

\section{CLUSTER PROPERTIES}

We use the membership data and the $R$-band images and X-ray contours shown in Figures 1 and 2 to either derive new centers for each cluster or confirm a previously reported center from the literature. These cluster centers are listed in Table 1. For most of the clusters, either a bright cluster member or the brightest cluster galaxy (BCG) is coincident with the approximate peak of the ICM. In these cases we adopt the coordinates of this galaxy for the cluster center, although for Abell 2163 the BCG candidate was not observed spectroscopically. Only Abell 3125 and Abell 3128 do not have obvious, bright cluster galaxies at their centers. Abell 3125 in fact does not have a diffuse ICM, while the ICM for Abell 3128 is double peaked. This pair of merging clusters have been extensively discussed and modeled by Rose et al. (2002), and we adopt their position for the center of Abell 3128, which is approximately midway between the peaks in the extended X-ray emission. For Abell 3125 we simply adopt the mean position of all of the confirmed members, which is within an arcminute of the position reported by Abell et al. (1989). Figure 3 shows the distribution of cluster members relative to the adopted centers along with the area subtended by the Chandra field of view.

\subsection{Velocity Dispersions and Membership}

We have used the spectroscopic data presented in Table 2 and literature membership data to determine the redshift, velocity dispersion, and redshift limits of each of these clusters. These 

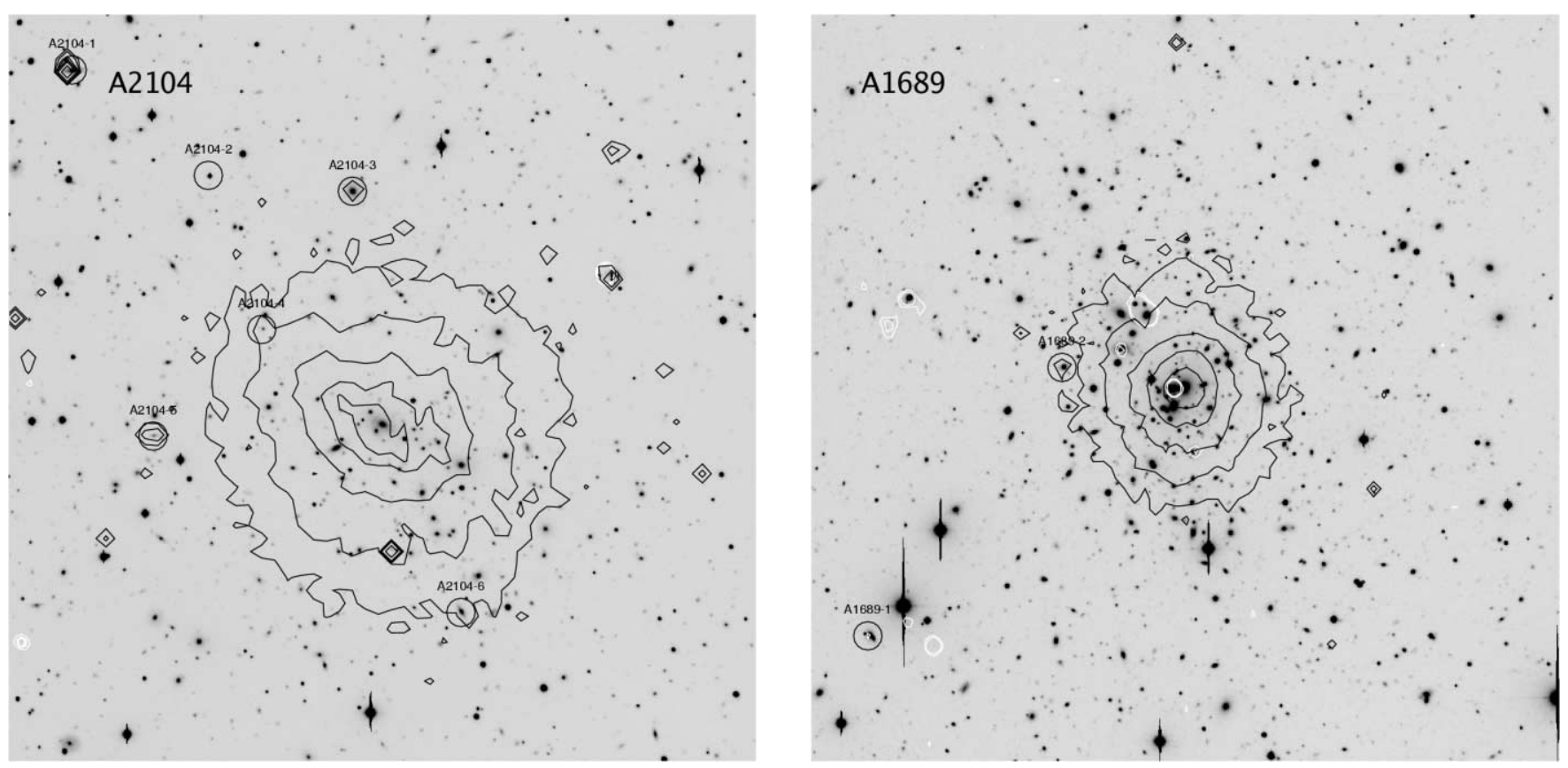

FIG. 1. $-R$-band images of Abell $2104(z=0.154)$ and Abell $1689(z=0.187)$ with Chandra X-ray (black contours $)$ and FIRST radio (white contours) data. Each image is $7^{\prime} \times 7^{\prime}$ on a side; north is up and east is to the left. These images only show the central region of our data where the X-ray emission is prominent.

values are presented in Table 1. We calculate these values with an implementation of the center and scale estimators described in Beers et al. (1990). For each cluster we combine our redshift measurements and any from the literature and calculate an approximate redshift and velocity dispersion using the biweight estimator for center and scale. We then removed galaxies more than $5 \sigma$ from the center and iterated to determine the mean redshift and velocity dispersion of each cluster. The adopted redshift range is $\pm 5 \sigma$ of the cluster mean. The uncertainty in the velocity dispersion is the $90 \%$ confidence limit calculated with the jackknife of the biweight estimator. Figure 4 shows the galaxy velocity distribution for each cluster over the range $\pm 3 \sigma$.

As noted above, there are literature measurements of multiple cluster members for all of these clusters except Abell 644 and Abell 2163. Rose et al. (2002) performed a detailed study of the merging clusters Abell 3125 and Abell 3128 with spectroscopic observations over a $2^{\circ}$ field and mapped out the complex velocity structure of this cluster pair. Our velocity measurements are broadly consistent with their results, although given the kinematic complexity of this system the derived velocity dispersions are unlikely to be a good measure of the cluster mass. As our observations have a substantially smaller field of view than the Rose et al. (2002) study and map only a small fraction of the physical extent of these clusters, the present observations are not as well suited to map the velocity structure in detail. Our measurements for Abell 2104, Abell 1689, MS 1008, and AC 114 are in good agreement with previous values. This is least surprising for Abell 2104, since we only have new redshifts for six X-ray counterparts to add to the study of Liang et al. (2000). AC 114 has the largest difference from the literature value. We measure a $15 \%$ larger velocity dispersion than the earlier estimate by Couch \& Sharples (1987) although a substantial number of new members have been identified since that work. Our value for Abell 1689 is comparable to the early measurement of Teague et al. (1990) and larger than the recent value of Czoske (2004), who measure $\sigma \approx 2100 \mathrm{~km} \mathrm{~s}^{-1}$ based on a larger sample of over 500 (unpublished) members; however, the difference between these values has no impact on our results below.

\subsection{Galaxy Colors and Spectral Properties}

In addition to changes in the mix of galaxy populations within clusters, such as the morphology-density relation (Dressler 1980), the mix of galaxy populations can also vary between clusters. One clear example of this is the Butcher-Oemler (BO) effect (Butcher \& Oemler 1978), the tendency for clusters to have larger blue galaxy fractions at higher redshift. Specifically, Butcher \& Oemler (1978) quantified the blue galaxy fraction in a cluster as the fraction of galaxies more than 0.2 mag bluer in rest-frame $B-V$ color. The color-magnitude diagrams presented in Figure 5 indicate that the confirmed cluster members (triangles) have a substantial variation in observed $B-R$ color. We calculate the BO galaxy fraction for each cluster as the fraction of all known members more luminous than $M_{R}<-20$ mag in our photometric data set. The number of $\mathrm{BO}$ galaxies and $\mathrm{BO}$ fraction are listed in Table 3. The highest fractions are measured for clusters with substantial star-forming galaxy populations (e.g., Abell 1689, AC 114). While the BO fraction is likely sensitive to potential biases such as the fraction of the cluster surveyed (e.g., fraction of the virial radius) and the limiting magnitude of the spectroscopy, the relative fractions provide a measure of the relative star formation activity from cluster to cluster in this sample. The difference between our measured BO fraction of $18 \%$ for AC 114 and the $29 \%$ measured by Couch \& Sharples (1987) provides a reasonable gauge of how sensitive this quantity is to measurement details.

We have also used our spectroscopic data for all of the clusters except Abell 2104 to determine the emission-line galaxy fraction for galaxies more luminous than $M_{R}<-20 \mathrm{mag}$. This provides a second measure of the frequency of star formation in cluster members, although this quantity is less reliable than the $\mathrm{BO}$ fraction because emission lines may be more difficult to detect in cluster members at higher redshift because the typical signal-to-noise ratio of the spectroscopy is lower. We simply classify any galaxy with an emission line as an emission-line galaxy, regardless of the strength of the line. In the vast majority of cases, the observed emission line is the [O II] $\lambda 3727$ doublet 
TABLE 2

Spectroscopic CAtalog

\begin{tabular}{|c|c|c|c|c|c|c|c|c|c|c|}
\hline $\begin{array}{l}\text { R.A. } \\
\text { (1) }\end{array}$ & $\begin{array}{l}\text { Decl. } \\
\text { (2) }\end{array}$ & $\begin{array}{c}z \\
(3)\end{array}$ & $\begin{array}{c}\text { Template } \\
\text { (4) }\end{array}$ & $\begin{array}{l}\text { Mask } \\
(5)\end{array}$ & $\begin{array}{l}\text { Select } \\
(6)\end{array}$ & $\begin{array}{r}R \\
\text { (7) }\end{array}$ & $\begin{array}{c}B-R \\
(8)\end{array}$ & $\begin{array}{c}V-R \\
(9)\end{array}$ & $\begin{array}{c}R-I \\
(10)\end{array}$ & $\begin{array}{l}\text { Lit ID } \\
(11)\end{array}$ \\
\hline 032745.35 & -532402.6 & 0.0599 & A & A3125a & $\mathrm{P}$ & $16.89(0.03)$ & $1.65(0.05)$ & $0.59(0.05)$ & $\ldots$ & \\
\hline 032733.96 & -532352.1 & 0.0626 & A & A3125a & $\mathrm{P}$ & $17.37(0.03)$ & $1.66(0.05)$ & $0.59(0.05)$ & $\ldots$ & \\
\hline 032722.03 & -532557.6 & 0.3950 & $\mathrm{E}$ & A3125a & $\mathrm{P}$ & $21.14(0.04)$ & $\ldots$ & $0.87(0.11)$ & $\ldots$ & \\
\hline 032752.60 & -532408.4 & 0.0614 & A & A3125a & $P$ & $15.93(0.03)$ & $1.77(0.05)$ & $0.66(0.05)$ & $\ldots$ & 2MASX J03275262-5324079 \\
\hline 032756.19 & $\begin{array}{lll}-53 & 23 & 18.4\end{array}$ & 0.4374 & E & $\mathrm{A} 3125 \mathrm{a}$ & $P$ & $20.88(0.03)$ & $1.40(0.08)$ & $0.68(0.05)$ & $\ldots$ & \\
\hline 032738.88 & -532604.5 & 0.597 & Q & A3125a & $\mathrm{X}$ & $19.91(0.03)$ & $0.35(0.05)$ & $0.14(0.05)$ & $\ldots$ & \\
\hline 032754.32 & -532151.1 & 0.84 & Q & A3125a & $\mathrm{X}$ & $20.80(0.03)$ & $0.64(0.05)$ & $0.28(0.05)$ & $\ldots$ & \\
\hline 032655.66 & -533158.8 & 0.3192 & A & A3125b & $\mathrm{P}$ & $19.18(0.03)$ & $2.71(0.07)$ & $0.97(0.05)$ & $\ldots$ & \\
\hline 032654.48 & -533147.9 & 0.3101 & E & A3125b & $\mathrm{P}$ & $\ldots$ & $\ldots$ & $\ldots$ & $\ldots$ & \\
\hline 032700.96 & -533136.6 & 0.0630 & E & A3125b & $\mathrm{P}$ & $19.91(0.03)$ & $1.09(0.05)$ & $0.38(0.05)$ & $\ldots$ & \\
\hline 032730.01 & $\begin{array}{llll}-53 & 31 & 04.4\end{array}$ & 0.2613 & A & A3125b & $\mathrm{P}$ & $19.72(0.03)$ & $1.55(0.05)$ & $0.56(0.05)$ & $\ldots$ & \\
\hline
\end{tabular}

NотеS.- Units of right ascension are hours, minutes, and seconds, and units of declination are degrees, arcminutes, and arcseconds. Spectroscopic catalog of all sources with successful redshift measurements. Cols. (1) and (2): Object right ascension and declination in J2000.0. Col. (3): Redshift. Col. (4): Best template match as emissionline galaxy (E), absorption-line galaxy (A), quasar (Q), or Galactic star (S). Col. (5): Mask identification. Col. (6): Target selection either as an X-ray source or from $R$-band photometry. Col. (7): $R$-band magnitude and uncertainty. Cols. (8)-(10): $B-R, V-R$, and $R-I$ color and uncertainty. Col. (11): Literature identification. Table 2 is published in its entirety in the electronic edition of the Astrophysical Journal. A portion is shown here for guidance regarding its form and content.

and nearly all of the emission-line galaxies appear to be starforming galaxies. The few exceptions are the small number of X-ray-identified AGNs with visible wavelength emission-line signatures. We do not spectroscopically identify any AGNs that do not have an X-ray counterpart. Both the BO fraction and emission-line galaxy fraction for these clusters are compared with the AGN fraction in $\S 5$ below.

\section{DISTRIBUTION OF CLUSTER AGNs}

Major mergers between gas-rich galaxies remain the standard paradigm for triggering high-luminosity quasars, although there is little direct evidence that such spectacular events are responsible for triggering lower luminosity AGNs as well (e.g., de Robertis et al. 1998; Schmitt 2001). However, in the standard picture of merger-driven fueling, the AGN luminosity diminishes gradually over up to a Gyr (e.g., Hopkins et al. 2005). Little evidence of a past merger may therefore be present during a more extended, low-luminosity phase.

As outlined above, major mergers between gas-rich galaxies should be substantially rarer in clusters of galaxies than in the field because the velocity dispersion is too high for major mergers and few galaxies are gas-rich. The most favorable region for major mergers to still occur in clusters is in the outskirts, which contain many galaxies falling into the cluster for the first time. These galaxies are primarily spirals and therefore are richer in cold gas. They are also often bound in low-velocity groups with velocity dispersions of a few hundred kilometers per second and consequently have a higher merger probability.

Previous studies have shown that there is more activity in the outskirts of clusters, such as a larger fraction of emission-line and poststarburst galaxies (e.g., Couch \& Sharples 1987; Fabricant et al. 1991; Fisher et al. 1998), although these studies did not specifically address the AGN distribution. In a study of 10 lowredshift clusters, Dressler et al. (1999) found that emission-line galaxies had a higher velocity dispersion than the more passive galaxies. The higher line-of-sight velocity dispersion implies that the emission-line galaxies are not yet virialized and remain on primarily radial orbits in the cluster potential.
Clusters of galaxies offer a unique opportunity to test if lowluminosity AGNs are primarily the result of the major mergers of gas-rich galaxies because these mergers are most likely to occur in the infalling population and the cluster crossing time is comparable to the predicted lifetime of the low-luminosity phase (e.g., Martini 2004). Low-luminosity AGNs triggered in infalling galaxies will therefore remain on primarily radial orbits after a Gyr, long after evidence of their violent past has faded. In the next subsections we investigate the kinematic and radial distribution of the cluster AGNs to determine if they may have recently entered the cluster potential.

\subsection{Velocity Distribution}

In Figure 4 we show the galaxy velocity distribution for each cluster and mark the locations of all of the X-ray sources with arrows and a dashed histogram. While there are a number of $\mathrm{X}$-ray sources several standard deviations from the mean cluster redshift, there are an insufficient number of sources per cluster to state if these outliers represent a significant fraction of the population.

To increase the AGN sample size for statistical analysis, we have combined the velocity distributions for all eight clusters. Because there is a substantial variation in the number of galaxies, number of X-ray sources, and velocity dispersions, we combined these clusters by first calculating the absolute value of the velocity offset of each galaxy from the cluster mean with respect to the cluster velocity dispersion. All eight clusters were then summed in these normalized coordinates, and in Figure 6 we show the cumulative distribution of the X-ray sources relative to the cluster galaxies without X-ray emission. We plot separate velocity distributions for all cluster AGNs more luminous than $L_{X}>$ $10^{41} \mathrm{ergs} \mathrm{s}^{-1}$ and all more luminous than $L_{\mathrm{X}}>10^{42} \mathrm{ergs} \mathrm{s}^{-1}$.

This figure demonstrates that the velocity distribution of cluster AGNs is essentially identical to the other cluster members. A Kolmogorov-Smirnov (K-S) test confirms this to be the case, specifically that there is a $68 \%$ and $96 \%$ probability that the $L_{\mathrm{X}}>10^{41} \mathrm{erg} \mathrm{s}^{-1}$ and $L_{\mathrm{X}}>10^{42} \mathrm{erg} \mathrm{s}^{-1}$ AGNs, respectively, are drawn from the same parent population as the cluster members 


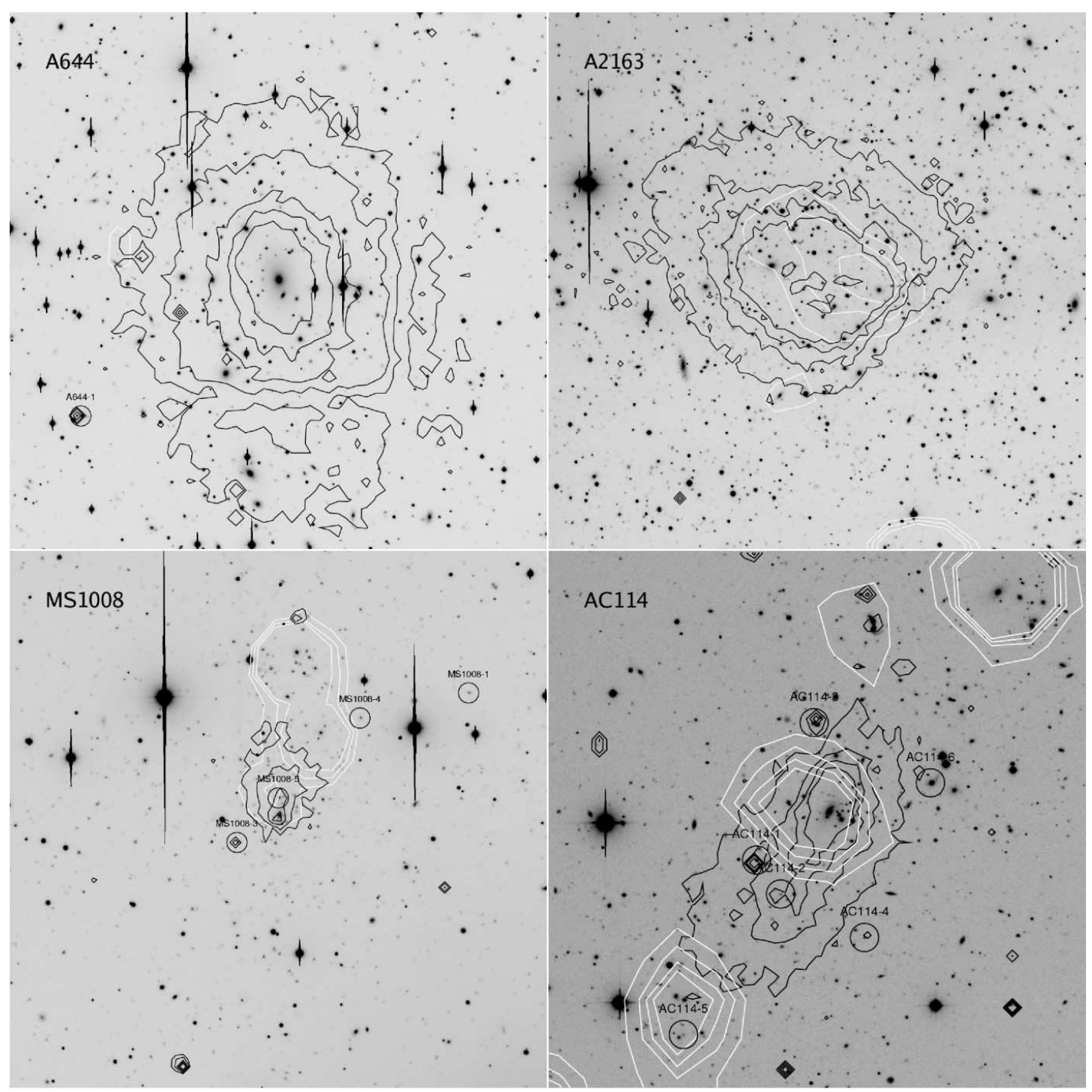

Fig. 2.- $R$-band images of Abell 644 (top leff; $z=0.070$ ), Abell 2163 (top right; $z=0.201$ ), MS 1008 (bottom left; $z=0.307$ ), and AC 114 (bottom right $z=0.315$ ) along with Chandra X-ray (black contours) and NVSS radio (white contours) data. Each image is $7^{\prime} \times 7^{\prime}$ on a side; north is up and east is to the left. As in Fig. 1, these images only show the central region of the cluster where the X-ray emission is prominent.

without X-ray counterparts (see Table 4). We therefore do not find evidence that the cluster AGNs are preferentially distributed on radial orbits, even if we exclude the merging clusters Abell $3125 / 3128$, unlike the case for emission-line galaxies in clusters (Dressler et al. 1999). This may indicate that nuclear activity in cluster galaxies can remain, or be reactivated, after the parent population has virialized in the cluster potential.

To test the sensitivity of our data to the known differences between cluster galaxy populations, we computed similar distributions between $\mathrm{BO}$ and non-BO galaxies, as well as between emission-line and absorption-line galaxies. This analysis showed that the $\mathrm{BO}$ galaxies have systematically larger velocity dispersions than the redder cluster members, as expected for a population on more radial orbits. The difference between emission-line and absorption-line cluster members was not statistically significant, although as noted previously, the emission-line classification is more susceptible to variations in the average signal-to-noise ratio than the color classification. The emission- and absorption-line samples used here are also only based on our spectroscopy, and not all known members, and therefore the sample is smaller than that used for the $\mathrm{BO}$ and red galaxy comparison.

\subsection{Radial Distribution}

To investigate the radial distribution of the AGNs relative to the cluster center, we have similarly combined all of the cluster sources shown in Figure 3 and plot the cumulative radial distribution of the AGNs and inactive cluster members in Figure 7. We have calculated this distribution as a function of projected physical distance from the cluster center in $\mathrm{Mpc}$ and as a function of projected fraction of $r_{200}$, where $r_{200}$ is the physical radius within which the mean density of a virialized cluster of the measured velocity dispersion exceeds the critical density at that redshift by a factor of 200 (e.g., Carlberg et al. 1997; Treu et al. 2003). This figure shows that the $L_{\mathrm{X}}>10^{41} \mathrm{erg} \mathrm{s}^{-1}$ cluster AGNs and the other cluster members have a similar radial distribution, but above $L_{\mathrm{X}}>10^{42} \mathrm{ergs} \mathrm{s}^{-1}$ the cluster $A G N$ s are more strongly concentrated. A K-S test confirms the visual impression of both panels of Figure 7. There are formally only $7 \%$ and $3 \%$ probabilities that the $L_{\mathrm{X}}>10^{42} \mathrm{erg} \mathrm{s}^{-1}$ AGNs are drawn from the same distribution in metric and virial radius, respectively (see Table 4). The probabilities are less than a factor of 2 higher when we cluster the merging clusters Abell 3125/3128. 


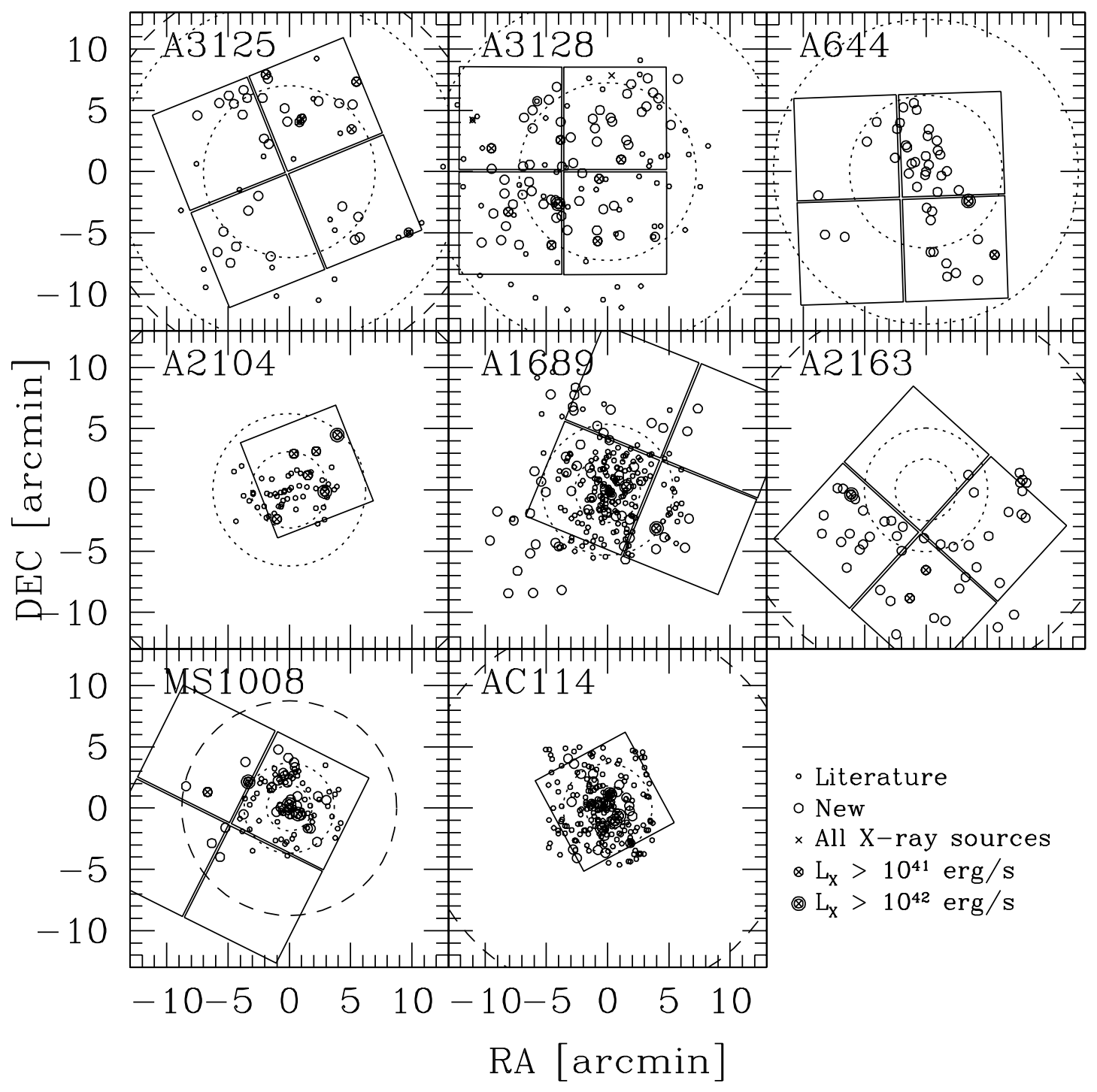

FIG. 3.-Positions of cluster members relative to the centers listed in Table 1. Members are marked with different symbols if their positions are from the literature (small circles), identified in the present study (large circles), and X-ray sources (crosses). Also shown are the field of view of the Chandra observations (solid black boxes) and circles of radius $0.5 \mathrm{Mpc}$ (smaller dotted circle), $1 \mathrm{Mpc}$ (larger dotted circle), and $r_{200}$ (large dashed circle), if the latter radius falls within the field of view shown. The axes on all panels are offsets in arcminutes from the cluster center. [See the electronic edition of the Journal for a color version of this figure.]

This result confirms the previous, purely statistical evidence for a concentration of AGNs in the centers of clusters (Ruderman \& Ebeling 2005; Dowsett 2005) with a spectroscopically confirmed sample. In a recent paper Lin \& Mohr (2007) found evidence that radio sources in clusters are also more centrally concentrated than typical cluster galaxies; however, we intriguingly do not find any evidence that the $\mathrm{X}$-ray and radio AGNs are in the same host galaxies. Comparison of our X-ray-selected AGNs and those in the radio study of Morrison et al. (2003) indicates that the two samples are nearly disjoint (see also the radio and X-ray contours in Figs. 1 and 2).

The summed radial distribution of cluster members is more sensitive to several potential biases than the summed velocity distribution. These biases include the physical area of the cluster surveyed for X-ray and other members and the relative physical sizes of the clusters. The first is important because the distributions will artificially appear different if the field of the $\mathrm{X}$-ray observations is larger or smaller than the field of view surveyed to obtain membership information for inactive galaxies. We have addressed this source of systematic error by only including our spectroscopically confirmed members in Figure 7, rather than all available members. Our spectroscopic sample is a suitable comparison sample because the $\mathrm{X}$-ray and other cluster galaxy candidates were observed with the same multislit masks and the AGNs and inactive galaxies in each cluster were targeted over the same radial distribution from the cluster center. We further refined the input catalog by only including confirmed cluster members that fall within the field of view of the Chandra observations.

The second potential bias is the different sizes of the clusters, both their physical size and projected angular extent relative to the Chandra field of view. For example, the ACIS-I camera on Chandra only encompasses the central $\sim 0.5 \mathrm{Mpc}$ of Abell 3125 and Abell 3128, while it encompasses up to $\sim 3 \mathrm{Mpc}$ from the center of MS 1008 (see Fig. 3). The redshift range of the sample therefore causes the higher redshift clusters to dominate the distribution at the largest distances from the cluster center. Similarly, only the low-mass cluster Abell 3125 and the higher redshift cluster MS 1008 provide substantial information about the distribution of cluster galaxies at more than half of $r_{200}$. The 

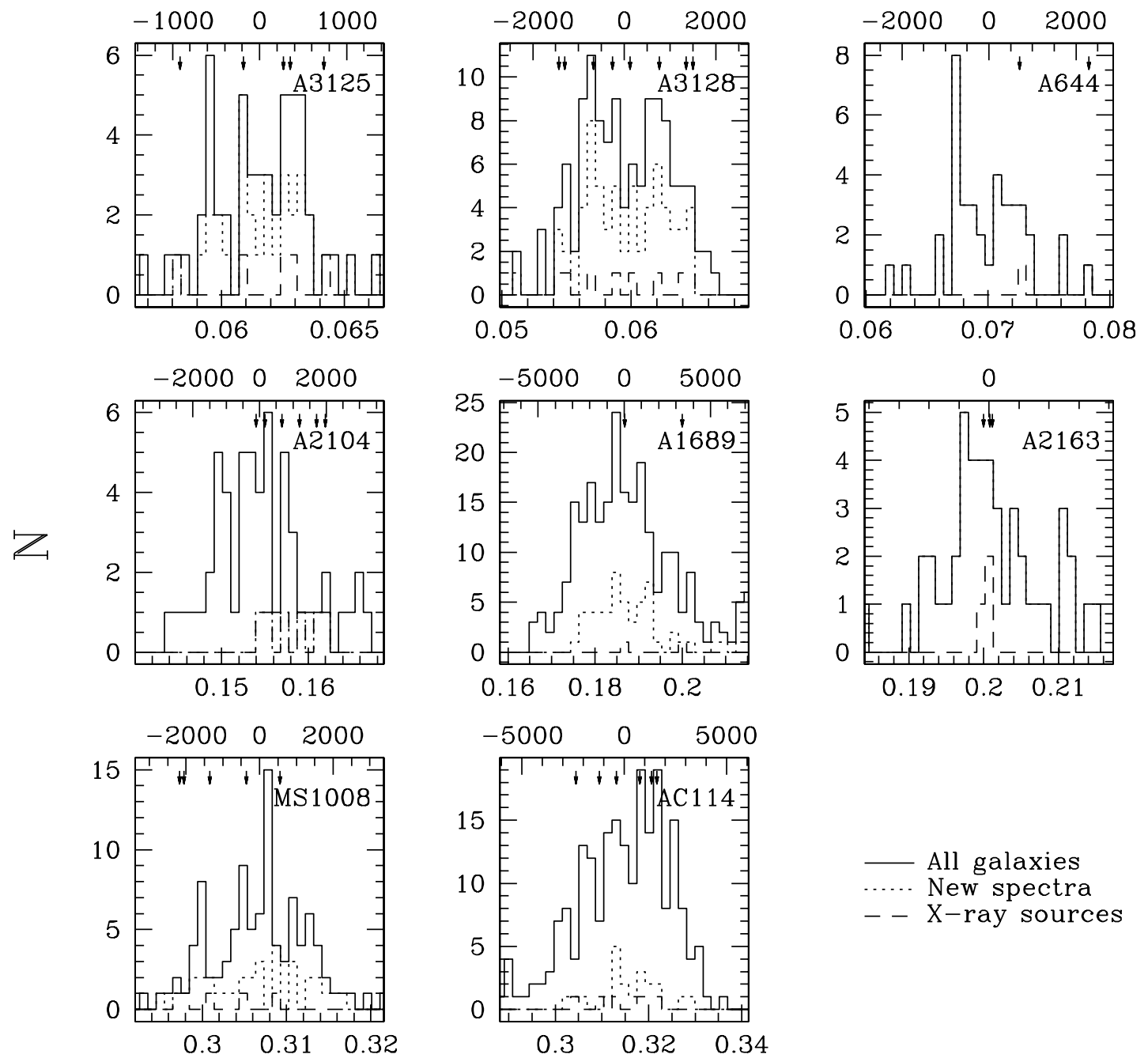

\section{Redshift and rest-frame $\mathrm{km} / \mathrm{s}$}

FIG. 4.-Velocity distributions for each cluster. Each panel displays the distribution of all galaxies within $3 \sigma$ of the cluster mean redshift (solid line), the subset of new members (dotted line), and the X-ray sources (dashed line) for a cluster field. The redshifts of individual X-ray sources are also marked (arrows). The bottom

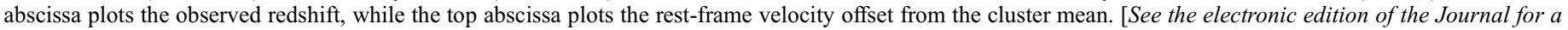
color version of this figure.]

virial scaling does not change the fact that the Chandra field of view samples a different fraction of the physical extent of different clusters.

A final potential bias on the radial distribution of X-ray sources is the detection efficiency of X-ray sources as a function of distance from the cluster center. The detection efficiency may be a function of clustercentric distance due to two effects: the higher background near the cluster center due to the ICM (see Figs. 1 and 2) and the degradation of the Chandra point-spread function (PSF) at larger off-axis angles may make it more difficult to detect sources. While the effect of the bright ICM would decrease the probability of detecting an X-ray source near the cluster core and therefore is opposite our observed trend, the larger Chandra $\mathrm{PSF}$ at larger off-axis angles could mimic the observed trend for $L_{\mathrm{X}}>10^{42} \mathrm{erg} \mathrm{s}^{-1}$ AGNs.

To quantify both of these potential biases on the apparent radial distribution, we performed a series of simulations with the MARX $^{1}$ package to quantify our detection efficiency as a func-

\footnotetext{
${ }^{1}$ See http://space.mit.edu/CXC/MARX.
}

tion of source luminosity and clustercentric distance. MARX is well suited to this task because it can generate a PSF that accounts for the off-axis angle, aspect solution, and spectral shape. The detection efficiency relative to the observed radial distribution of all members with $M_{R}<-20$ mag and no X-ray detection is the most relevant quantity because our comparison is directly with these galaxies. We therefore used MARX to add an artificial $\mathrm{X}$-ray point source at the location of every cluster member without an X-ray source. This source was assigned a $\Gamma=1.7$ spectral shape and the total counts corresponding to a given luminosity at the redshift of the particular cluster. The fraction of these sources recovered with the same wavdetect parameters used in Paper I then provides a measurement of the detection efficiency. We performed this exercise for point sources with total counts corresponding to a source with $L_{\mathrm{X}}=10^{41}$ and $10^{42} \mathrm{ergs} \mathrm{s}^{-1}$ for each cluster and then calculated the detection efficiency as a function of clustercentric distance in both Mpc and fraction of the virial radius. From this approach we find that the detection efficiency varies by less than $20 \%$ out to $1.5 \mathrm{Mpc}$ and $0.5 r_{200}$, after which the results begin to be compromised by small number statistics 


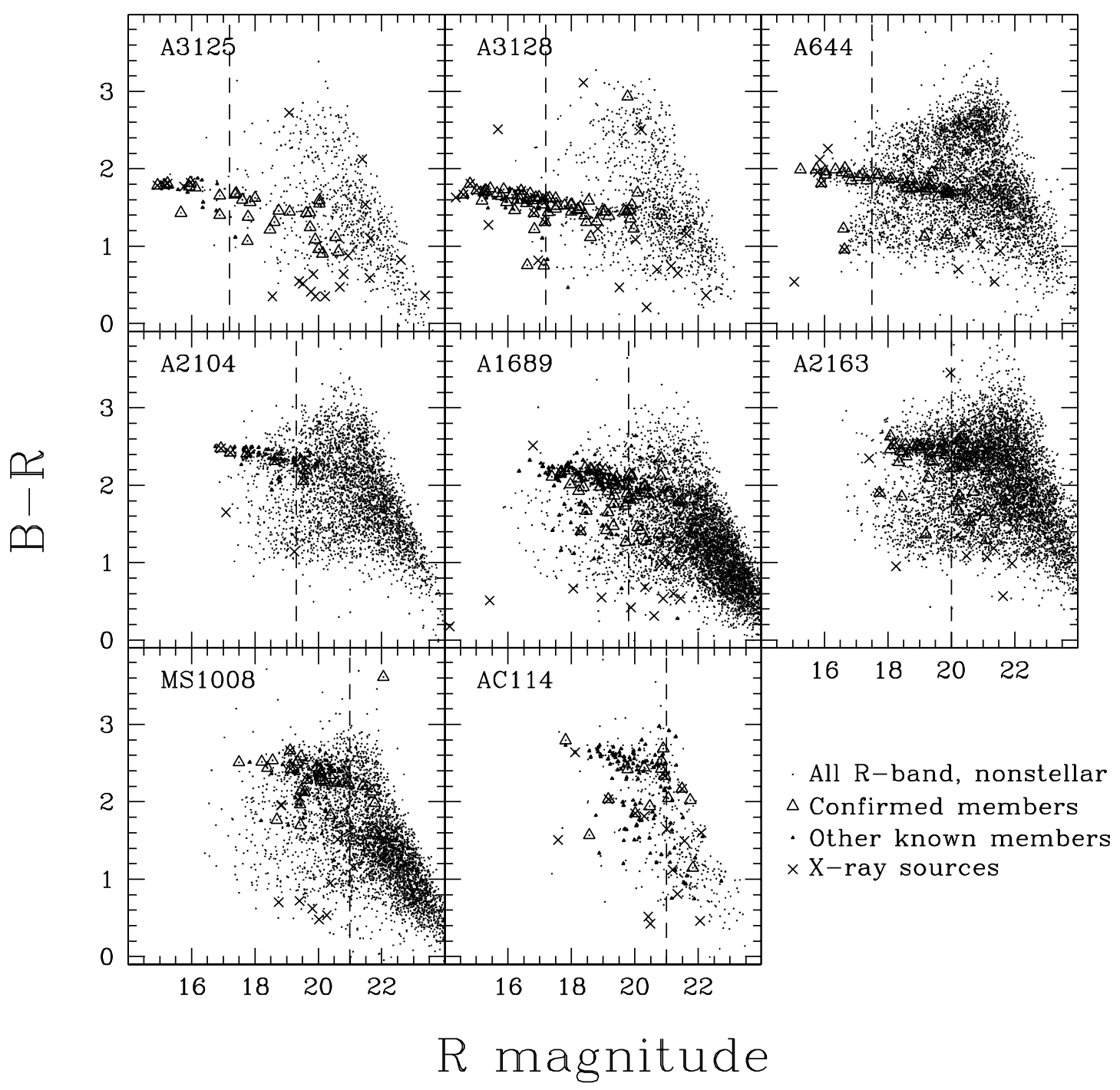

FIG. 5.-Color-magnitude diagrams for the eight cluster fields. All sources in our $R$-band catalog with measured $B-R$ colors and $R$-band stellarity less than 0.9 are shown. Confirmed members from Table 2 trace the color-magnitude relation of the passively evolved galaxies in each cluster (open triangles). Other members from the literature (small triangles) and X-ray sources (crosses) are also marked. The vertical dashed line marks the apparent magnitude that corresponds to a rest-frame $M_{R}=-20 \mathrm{mag}$ for each cluster. [See the electronic edition of the Journal for a color version of this figure.]

TABLE 3

Cluster Galaxy Population Fractions

\begin{tabular}{|c|c|c|c|c|}
\hline $\begin{array}{l}\text { Cluster } \\
\text { (1) }\end{array}$ & $\begin{array}{c}N_{\text {BO }} \\
(2)\end{array}$ & $\begin{array}{c}f_{\mathrm{BO}} \\
(3)\end{array}$ & $\begin{array}{l}N_{e} \\
\text { (4) }\end{array}$ & $\begin{array}{l}f_{e} \\
(5)\end{array}$ \\
\hline Abell $3125 \ldots \ldots \ldots \ldots \ldots$ & 2 & 0.11 & 4 & 0.44 \\
\hline Abell 3128 & 5 & 0.07 & 7 & 0.21 \\
\hline Abell 644 & 2 & 0.13 & 1 & 0.06 \\
\hline Abell 2104 & 3 & 0.09 & $\ldots$ & $\ldots$ \\
\hline Abell 1689 & 31 & 0.25 & 8 & 0.24 \\
\hline Abell 2163 & 3 & 0.12 & 3 & 0.11 \\
\hline MS 1008 & 18 & 0.31 & 4 & 0.16 \\
\hline AC 114 & 25 & 0.26 & 2 & 0.18 \\
\hline
\end{tabular}

Notes.- The fraction of $\mathrm{BO}$ and emission-line galaxies in each cluster that are brighter than a fixed rest-frame absolute magnitude of $M_{R}=-20 \mathrm{mag}$. For each cluster listed in col. (1) we list the total number of BO galaxies that are known cluster members in col. (2) and their fraction of the total cluster galaxy population above that luminosity in col. (3). We present the total number of emission-line galaxies and the emission-line galaxy fraction in cols. (4) and (5), although this quantity is only calculated from galaxies we observed spectroscopically. We do not have sufficient data to calculate this quantity for Abell 2104. See $\S 3.2$ for further details. (few cluster members). This is true for both $10^{41}$ and $10^{42} \mathrm{erg} \mathrm{s}^{-1}$ sources, and we therefore conclude that the observed trend with radius is real and not an artifact of either bias. We note further that this approach is somewhat pessimistic as it assumes that all of the point sources are at the luminosity limit, rather than at or above it, but it nevertheless provides a relative measure of the detection probability.

As in $\S 4.1$, we compared the radial distributions of photometrically (BO or red) and spectroscopically (emission line or absorption line) defined subsamples to test the sensitivity of these data to other known relations between cluster populations. This analysis showed that the red galaxies are more centrally concentrated than the BO population, as expected. We also found no statistically significant difference between the emission-line and absorption-line populations, which we again attribute to the smaller size of the subsamples input to this analysis.

\subsection{Association with Cluster Substructure}

If cluster AGNs are preferentially associated with infalling groups of galaxies, or other structures within clusters, then they may trace local deviations from the mean cluster kinematics. 


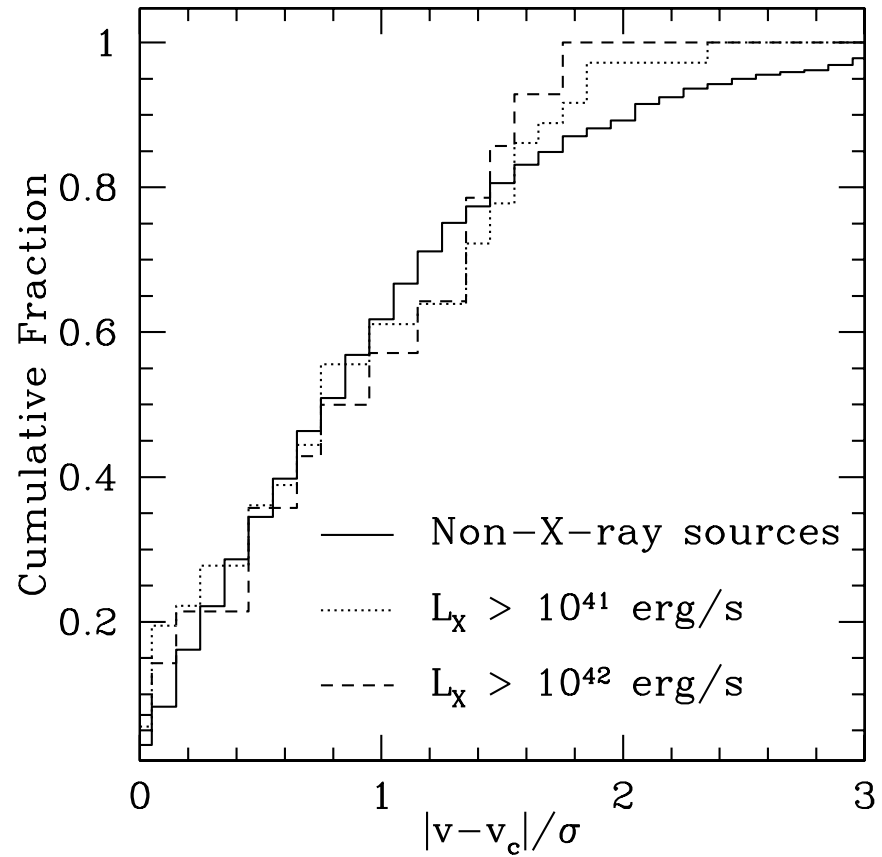

FIG. 6.-Cumulative, normalized velocity distribution of cluster X-ray sources relative to each cluster's mean redshift. The distributions of the AGNs more luminous than $L_{\mathrm{X}}>10^{41} \mathrm{ergs}^{-1}$ (dotted line), more luminous than $L_{\mathrm{X}}>10^{42} \mathrm{ergs} \mathrm{s}^{-1}$ (dashed line), and the inactive galaxies (solid line) are statistically indistinguishable. The absolute value of the velocity offset of each galaxy from the cluster mean has been normalized to the cluster's velocity dispersion. See $\S 4.1$ for further details. [See the electronic edition of the Journal for a color version of this figure.]

Dressler \& Shectman (1988) developed a substructure test to identify deviations in the cluster velocity field, where the substructure parameter $\delta$ is defined as

$$
\delta^{2}=\left(11 / \sigma^{2}\right)\left[\left(\bar{v}_{\text {local }}-\bar{v}\right)^{2}+\left(\sigma_{\text {local }}-\sigma\right)^{2}\right]
$$

and the local values of the radial velocity $\bar{v}_{\text {local }}$ and velocity dispersion $\sigma_{\text {local }}$ are calculated from the radial velocities of each galaxy and its 10 nearest neighbors. Larger values of $\delta$ correspond to larger local deviations from the cluster kinematics. Figure 8 plots all of the cluster members shown in Figure 3 with open circles scaled by this substructure measure. One minor change from the method of Dressler \& Shectman (1988) is that we calculate these quantities with the biweight estimator described previously, rather than assume a Gaussian distribution.

This analysis indicates that Abell 3125 and Abell 3128 have the most substantial substructure. Dressler \& Shectman (1988) note that the sum of the $\delta$-values for all cluster members is a measure of how much substructure is present, and a simple way to characterize this value for a given cluster is via Monte Carlo simulations with random reshuffling of the measured velocities among the measured positions. We have generated 10,000 realizations with random redistributions of the measured velocities among the measured galaxy positions and calculated the probability of obtaining the measured sum of the $\delta$-values or larger. These values are listed in Table 1 and indicate that Abell 3125 and Abell 3128 indeed have significant substructure, which is not surprising for a merging pair of clusters, while the remaining clusters do not have substantial substructure.

The substructure parameter can also be used to characterize the extent that a particular cluster population is associated with
TABLE 4

K-S Test Results

\begin{tabular}{|c|c|c|}
\hline $\begin{array}{l}\text { Distribution Type } \\
\text { (1) }\end{array}$ & $\begin{array}{l}\text { Sample Limits } \\
\text { (2) }\end{array}$ & $\begin{array}{l}\text { K-S } \\
\text { (3) }\end{array}$ \\
\hline \multirow[t]{4}{*}{ Velocity distribution } & $L_{\mathrm{X}}>10^{41}$ & 0.683 \\
\hline & $L_{\mathrm{X}}>10^{42}$ & 0.955 \\
\hline & $L_{\mathrm{X}}>10^{41}-\mathrm{A} 3125 / 8$ & 0.388 \\
\hline & $L_{\mathrm{X}}>10^{42}-\mathrm{A} 3125 / 8$ & 0.967 \\
\hline \multirow[t]{4}{*}{ 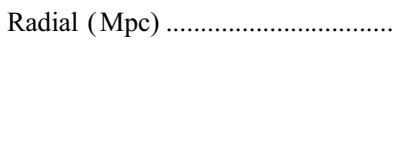 } & $L_{\mathrm{X}}>10^{41}$ & 0.743 \\
\hline & $L_{\mathrm{X}}>10^{42}$ & 0.069 \\
\hline & $L_{\mathrm{X}}>10^{41}-\mathrm{A} 3125 / 8$ & 0.950 \\
\hline & $L_{\mathrm{X}}>10^{42}-\mathrm{A} 3125 / 8$ & 0.128 \\
\hline \multirow[t]{4}{*}{ 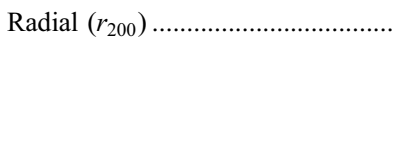 } & $L_{\mathrm{X}}>10^{41}$ & 0.989 \\
\hline & $L_{\mathrm{X}}>10^{42}$ & 0.028 \\
\hline & $L_{\mathrm{X}}>10^{41}-\mathrm{A} 3125 / 8$ & 0.513 \\
\hline & $L_{\mathrm{X}}>10^{42}-\mathrm{A} 3125 / 8$ & 0.051 \\
\hline \multirow[t]{4}{*}{ Substructure. } & $L_{\mathrm{X}}>10^{41}$ & 0.616 \\
\hline & $L_{\mathrm{X}}>10^{42}$ & 0.606 \\
\hline & $L_{\mathrm{X}}>10^{41}-\mathrm{A} 3125 / 8$ & 0.302 \\
\hline & $L_{\mathrm{X}}>10^{42}-\mathrm{A} 3125 / 8$ & 0.649 \\
\hline
\end{tabular}

Notes.-Results of the K-S tests. Cols. (1) and (2) describe the input sample of X-ray sources; in all cases the comparison sample comprises the cluster members without X-ray emission. Col. (3) lists the probability that the X-ray sources are drawn from a different distribution. Sample limits marked " - A3125/8" did not include these two clusters. See $\S 4$ for further details.

substructure. We have calculated the distribution of $\delta$-values for the X-ray sources in all eight clusters relative to the other cluster members and plot this distribution in Figure 9. There is good agreement between the distribution of the $L_{\mathrm{X}}>10^{41} \mathrm{erg} \mathrm{s}^{-1}$, $L_{\mathrm{X}}>10^{42} \mathrm{erg} \mathrm{s}^{-1}$, and cluster members without X-ray counterparts, and this indicates that the cluster AGNs are not more likely to be associated with substructure in clusters, at least on the scales this parameter is sensitive to (a galaxy and its 10 nearest neighbors). As Abell 3125 and Abell 3128 have substantial substructure, we repeated this exercise without these two clusters and still obtained consistent distributions. Results from K-S tests of all of these distributions are listed in Table 4.

\section{RELATION OF AGNS TO CLUSTER PROPERTIES}

In the previous two sections we have analyzed the properties of the clusters and the distribution of the AGNs within the clusters. In the present section we discuss the completeness of our survey of each cluster and investigate potential correlations between the AGN fraction and the properties of their host cluster.

\subsection{Completeness}

In Paper I we calculated the AGN fraction in clusters of galaxies and defined the AGN fraction as the fraction of galaxies more luminous than a rest-frame $M_{R}=-20 \mathrm{mag}$ with X-ray counterparts more luminous than $L_{\mathrm{X}}=10^{41} \mathrm{ergs} \mathrm{s}^{-1}$ in the broad X-ray band, or $f_{A}\left(M_{R}<-20 ; L_{\mathrm{X}}>10^{41}\right)=5 \% \pm 1.5 \%$. The quoted uncertainty in this value was only a Poisson estimate and did not take into account potential systematic uncertainties, such as the estimate of the total cluster galaxy population. The determination of the numerator in the AGN fraction requires redshift measurements for all X-ray counterparts brighter than the apparent magnitude of a cluster member with this absolute magnitude. As we discussed in $\S 2$, our spectra are complete to the appropriate apparent magnitude limit for each cluster. Measurement of the denominator requires a much larger number of spectra because most galaxies to the requisite apparent magnitude limit in the Chandra field of view are not detected X-ray sources. For all but the lowest redshift clusters, these data are 


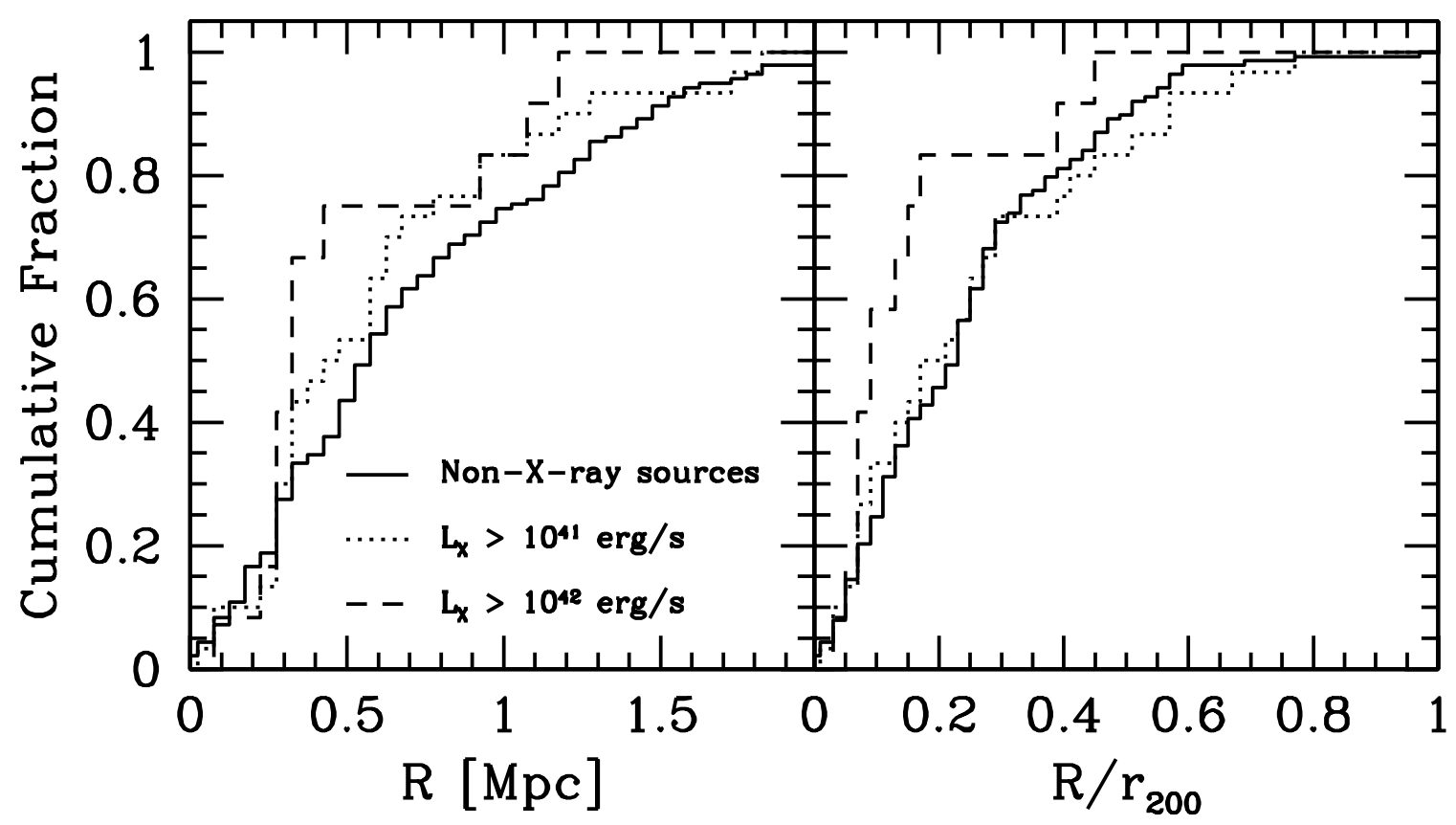

FIG. 7.-Cumulative, normalized radial distributions relative to each cluster's center in projected Mpc (left) and in units of $r_{200}(r i g h t)$. The distribution of the AGNs more luminous than $L_{\mathrm{X}}>10^{42} \mathrm{ergs} \mathrm{s}^{-1}$ (dashed line) is substantially more centrally concentrated than the inactive galaxies (solid line) in both radial distributions. The distribution of the AGNs more luminous than $L_{\mathrm{X}}>10^{41} \mathrm{ergs} \mathrm{s}^{-1}$ (dotted line) is comparable to the inactive galaxies. The radial distribution is calculated from the projected distance of each galaxy from the cluster center. Larger separations are sampled by the more distant clusters. See $\S 4.2$ for further details. [See the electronic edition of the Journal for a color version of this figure.]

substantially incomplete and we have to sum the known members with an estimate of the additional cluster members without redshifts to estimate the total number of cluster members brighter than $M_{R}=-20$ mag. As noted in Paper I, we can quantify this completeness with photometric observations of known cluster members, known nonmembers, and galaxies without spectra. Here we estimate the total number of cluster members without spectra by calculating the fraction of galaxies with spectra that are cluster members as a function of $B-R$ color, rather than adopt a color-independent completeness estimate as in Paper I. This color-dependent completeness estimate takes into account the observation that galaxies with similar colors to observed cluster members are more likely to be cluster members than those with dramatically different colors, but it still accounts for a potential population of bluer or $\mathrm{BO}$ galaxies if such a population has been spectroscopically confirmed. A disadvantage of this approach is that the completeness estimate is sensitive to the number of members and nonmembers with spectra at a given color. We have increased the number of galaxies with spectra at a given color by also including known members and nonmembers from the literature for which we have photometry, although they fall outside of the Chandra field of view. However, these additional data are only used to provide an improved completeness fraction as a function of color; the total number of cluster members is estimated only from galaxies that fall within the Chandra field of view.

In Table 5 we use the results of this completeness calculation to obtain an estimate of the total number of cluster members more luminous than $M_{R}=-20$ mag. For each cluster we list both the number of known members from our data and the literature that fall within the Chandra field of view and our estimate of the total cluster galaxy population. We then calculate the AGN fraction for these clusters with three different cuts in X-ray luminosity: (1) the fraction of AGNs if all of the X-ray sources in galaxies with $M_{R}<-20$ mag identified in Paper I are AGNs,
(2) the AGN fraction for only those X-ray sources above a luminosity of $L_{\mathrm{X}}=10^{41} \mathrm{ergs} \mathrm{s}^{-1}$, and (3) the AGN fraction for those above $L_{\mathrm{X}}=10^{42} \mathrm{ergs} \mathrm{s}^{-1}$. The second of these three limits, namely, $M_{R}<-20$ mag and $L_{\mathrm{X}}>10^{41} \mathrm{ergs} \mathrm{s}^{-1}$, corresponds to the AGN fraction quoted in Paper I. Here we derive $f_{A}\left(M_{R}<\right.$ $\left.-20 ; L_{\mathrm{X}}>10^{41}\right)=5 \%$, in excellent agreement with our calculation from Paper I. This correction provides a better estimate of the membership fraction for galaxies observed to be redder and bluer than typical, known members. The X-ray luminosity threshold was chosen previously because it is rare for other potential sources of X-ray emission, most notably LMXBs and hot, gaseous halos, to produce such high X-ray luminosities. However, as noted in Paper I, the disadvantage of this threshold is that the X-ray observations of Abell 1689 and MS 1008 are not quite sensitive to this luminosity limit and therefore some AGNs may be missed and the AGN fraction underestimated. We have also calculated the AGN fraction for a factor of 10 higher X-ray luminosity and find $f_{A}\left(M_{R}<-20 ; L_{\mathrm{X}}>10^{42}\right)=1 \%$. All of our $\mathrm{X}$-ray observations are sensitive to such luminous sources. Galaxies that can produce such luminous X-ray emission by mechanisms other than black hole accretion are also much rarer than AGNs.

The AGN fraction may also be a function of host galaxy luminosity, in addition to X-ray luminosity. In our original work on Abell 2104 (Martini et al. 2002), we noted that two of the three brightest cluster members were AGNs. From inspection of Figure 5, a similar tendency is apparent at the bright end of the color-magnitude relation in Abell 3125, Abell 3128, and Abell 2163. In fact, approximately half (19) of the AGNs more luminous than $L_{\mathrm{X}}>10^{41} \mathrm{ergs} \mathrm{s}^{-1}$ are in host galaxies more luminous than $M_{R}<-21.3 \mathrm{mag}$, and for these galaxies we find $f_{A}\left(M_{R}<-21.3 ; L_{\mathrm{X}}>10^{41}\right)=9.8 \%$. This absolute magnitude limit was chosen to correspond to the luminosity threshold of Sun et al. (2007), who find nine AGNs with $L_{X}>$ $10^{41} \mathrm{ergs} \mathrm{s}^{-1}$ in 163 galaxies in a sample of $z=0.01-0.05$ 


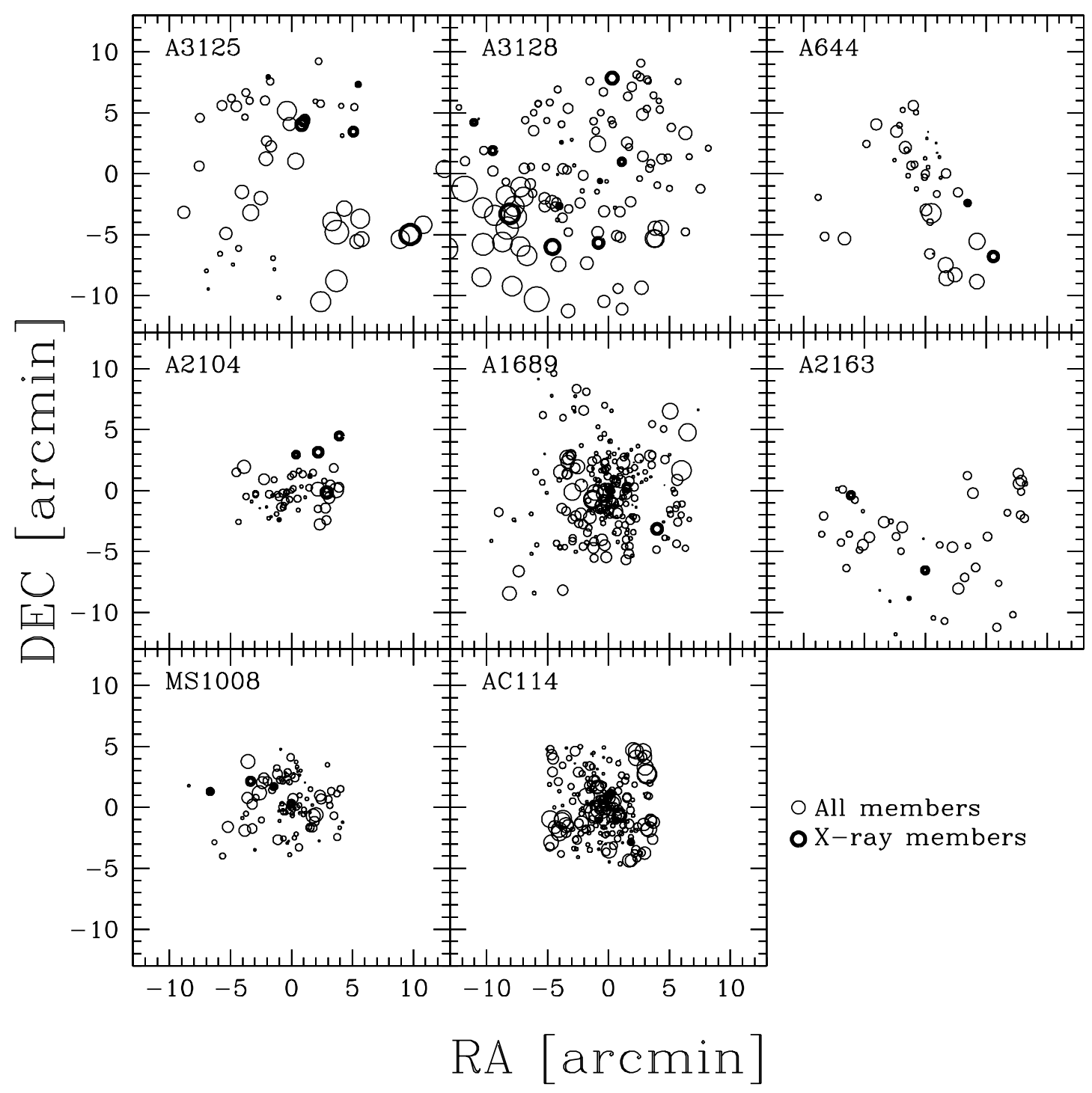

Fig. 8. - Cluster substructure calculated from known cluster members. Positions of all cluster members (thin circles) and X-ray members (thick circles) are relative to the centers listed in Table 1 . The size of each circle is scaled by the value of the substructure parameter $\delta$ for each source. The axes on all panels are offsets in arcminutes from the cluster center. [See the electronic edition of the Journal for a color version of this figure.]

clusters, or $f_{A}\left(M_{R}<-21.3 ; L_{\mathrm{X}}>10^{41}\right)=5 \%$. While this value is a factor of 2 below our value, the differences may be due to variations in the AGN fraction between cluster samples (e.g., different redshift distributions), and we discuss this point further in $\S 5.2$.

An important caveat to these estimates is that they are estimates of the AGN fraction within the field of view of the Chandra ACIS-I or ACIS-S detectors. These detectors have an approximate field of view of $16.9^{\prime} \times 16.9^{\prime}$ and $8.3^{\prime} \times 8.3^{\prime}$, respectively. These two angular sizes, combined with the redshift distribution of our sample and the range in cluster sizes (see, e.g., the $r_{200}$ value in Table 1), correspond to substantial variations in the fraction of each cluster galaxy population surveyed. This point is illustrated with Figure 3, which includes circles that mark the $r_{200}$ radius. The Chandra field of view approximately covers out to the $r_{200}$ radius for only MS 1008 , while for many of the remaining clusters it is even larger than the $25 \operatorname{arcmin}^{2}$ panels shown for each cluster. Our measurements of the AGN fraction for each cluster are therefore necessarily measurements dominated by the center of the clus- ter for nearly all cases. However, the absence of a strong radial trend in the AGN distribution for the more common $L_{\mathrm{X}}>$ $10^{41} \mathrm{erg} \mathrm{s}^{-1}$ sources shown in Figure 7 suggests that the ratio of AGNs to inactive galaxies may not be a strong function of position within the cluster. The AGN fraction may therefore be relatively well determined, even if the census of the total cluster AGN population is incomplete. We also note that for several clusters our ground-based images do not encompass the entire Chandra field, and in fact some of the X-ray sources identified in Abell 3125 and Abell 3128 are from literature positions and redshifts, but the measurement of the AGN fraction remains relatively robust in the absence of strong radial gradients.

We have assumed that each cluster of galaxies has the same, intrinsic AGN fraction (and the same X-ray luminosity function) and treated each cluster as an independent measure of the AGN fraction. This yields an average fraction of $5 \%$ for $L_{\mathrm{X}}>$ $10^{41} \mathrm{ergs} \mathrm{s}^{-1}$ and $1 \%$ for $L_{\mathrm{X}}>10^{42} \mathrm{ergs} \mathrm{s}^{-1}$. If every cluster galaxy had an equal probability of hosting an AGN, an alternate way to calculate the AGN fraction in clusters of galaxies would be to treat each cluster galaxy as an independent measure of 


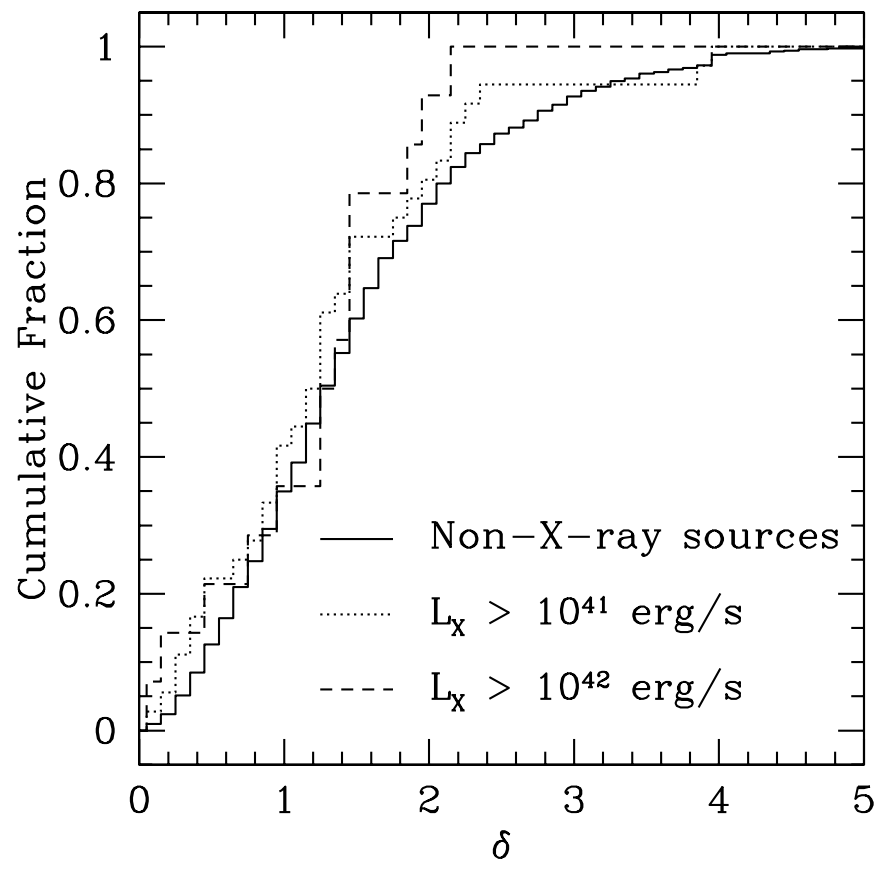

FIG. 9.-Cumulative distribution of the substructure parameter $\delta$ for X-ray sources more luminous than $L_{\mathrm{X}}>10^{41} \mathrm{ergs} \mathrm{s}^{-1}$ (dotted line), more luminous than $L_{\mathrm{X}}>10^{42} \mathrm{ergs} \mathrm{s}^{-1}$ (dashed line), and inactive cluster members (solid line). Larger $\delta$ corresponds to a larger local deviation from the mean cluster kinematics. [See the electronic edition of the Journal for a color version of this figure.]

the AGN fraction, rather than each cluster. This yields an AGN fraction of $2.2 \%$ for $L_{\mathrm{X}}>10^{41} \mathrm{ergs} \mathrm{\textrm {s } ^ { - 1 }}$ and $0.9 \%$ for $L_{\mathrm{X}}>$ $10^{42} \mathrm{ergs} \mathrm{s}^{-1}$. The value for $L_{\mathrm{X}}>10^{41} \mathrm{erg} \mathrm{s}^{-1}$ sources is substantially lower than the average of the cluster AGN fractions and provides evidence that the AGN fraction may vary from cluster to cluster.

\subsection{Evidence for Intrinsic Variation}

We now investigate variations in the AGN fraction from cluster to cluster and consider if these variations are consistent with
Poisson fluctuations and systematic errors in the cluster membership. If we ignore potential systematic errors in the completeness correction, the AGN fractions in these eight clusters are inconsistent with Poisson fluctuations with greater than $95 \%$ confidence for $L_{\mathrm{X}}>10^{41} \mathrm{ergs} \mathrm{s}^{-1}$; there is no evidence for variations in the AGN fraction for the much smaller population with $L_{\mathrm{X}}>10^{42} \mathrm{ergs} \mathrm{s}^{-1}$. The dominant source of systematic error in the AGN fraction is the estimate for the number of cluster galaxies in the Chandra field of view. This correction is more important for clusters that have a larger number of galaxies within the field of view of the Chandra observation and those clusters that have less membership data. The ratio of the estimated to confirmed cluster galaxy populations brighter than $M_{R}<-20 \mathrm{mag}$ in Table 5 provides the size of the correction and varies from $\sim 1$ for Abell 3128 to $\sim 10$ for Abell 2163 with a median of $\sim 2.5$. The ratio of the number of AGNs to the number of confirmed members provides a strong upper limit to the AGN fraction in each cluster. If we use this larger value for the clusters with the smallest AGN fraction, rather than the value listed in Table 5, we still find that the AGN fraction in these clusters is inconsistent with the values derived for the lower redshift clusters with small systematic errors. For example, the well-studied, highredshift cluster AC 114 has an AGN fraction of 5.2\% based on confirmed members alone, and this upper limit is still inconsistent with the AGN fraction in clusters such as Abell 3125 and Abell 3128 that have small completeness corrections. The variation in the AGN fraction from cluster to cluster also does not seem to be due to radial dependence in the AGN fraction, as Figure 7 indicates that there is not a difference between the distributions of the $L_{\mathrm{X}}>10^{41} \mathrm{erg} \mathrm{s}^{-1}$ population and other cluster members.

\subsection{Correlations with Cluster Properties}

As there is evidence for variation in the AGN fraction from cluster to cluster, we compare the AGN fraction from each cluster with four properties that may influence the AGN fraction: cluster redshift, cluster velocity dispersion, amount of cluster substructure, and the fraction of BO galaxies in the cluster. Simple arguments about the nature of AGN fueling and known

TABLE 5

Completeness and AGN Fractions

\begin{tabular}{|c|c|c|c|c|c|c|c|c|c|}
\hline \multirow[b]{2}{*}{$\begin{array}{c}\text { Cluster Name } \\
\text { (1) }\end{array}$} & \multirow[b]{2}{*}{$\begin{array}{l}N_{\mathrm{X}} \\
(2)\end{array}$} & \multicolumn{3}{|c|}{$N_{\mathrm{X}}(<-20 \mathrm{mag})$} & \multicolumn{2}{|c|}{ Membership } & \multicolumn{3}{|c|}{$f_{A}$} \\
\hline & & $\begin{array}{l}\text { All } \\
(3)\end{array}$ & $\begin{array}{c}>10^{41} \\
(4)\end{array}$ & $\begin{array}{c}>10^{42} \\
(5)\end{array}$ & $\begin{array}{l}\text { Confirmed } \\
\text { (6) }\end{array}$ & $\begin{array}{l}\text { Estimated } \\
\text { (7) }\end{array}$ & $\begin{array}{l}\text { All } \\
(8)\end{array}$ & $\begin{array}{c}>10^{41} \\
(9)\end{array}$ & $\begin{array}{r}>10^{42} \\
(10)\end{array}$ \\
\hline 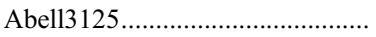 & 6 & 5 & 4 & 0 & 18 & 28 & 0.179 & 0.143 & 0.000 \\
\hline 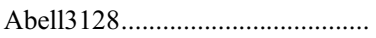 & 10 & 10 & 7 & 1 & 54 & 63 & 0.159 & 0.111 & 0.016 \\
\hline 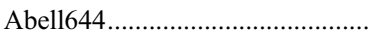 & 2 & 2 & 2 & 1 & 15 & 75 & 0.027 & 0.027 & 0.013 \\
\hline 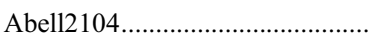 & 6 & 4 & 4 & 1 & 33 & 62 & 0.065 & 0.065 & 0.016 \\
\hline 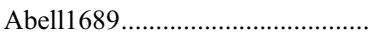 & 2 & 2 & 2 & 2 & 110 & 337 & 0.006 & 0.006 & 0.006 \\
\hline Abell2163...... & 3 & 2 & 2 & 1 & 25 & 262 & 0.008 & 0.008 & 0.004 \\
\hline 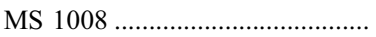 & 5 & 4 & 4 & 3 & 75 & 346 & 0.012 & 0.012 & 0.009 \\
\hline 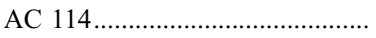 & 6 & 5 & 5 & 3 & 95 & 204 & 0.025 & 0.025 & 0.015 \\
\hline Average & $\ldots$ & $\ldots$ & $\ldots$ & $\ldots$ & $\ldots$ & $\ldots$ & 0.060 & 0.049 & 0.010 \\
\hline Sum & 40 & 35 & 30 & 12 & 425 & 1377 & 0.025 & 0.022 & 0.009 \\
\hline
\end{tabular}

Notes. - Completeness estimates and AGN fractions for galaxies more luminous than $M_{R}=-20$ mag and different X-ray luminosity cuts. Col. (1): Cluster name. Col. (2): Number of X-ray sources in the cluster regardless of the host galaxy luminosity. Col. (3): Number of X-ray sources in hosts more luminous than $M_{R}=-20$ mag. Col. (4): Number of X-ray sources more luminous than $L_{\mathrm{X}}=10^{41} \mathrm{ergs} \mathrm{s}^{-1}$ in the broad X-ray band. Col. (5): Number of X-ray sources more luminous than $L_{\mathrm{X}}=10^{42} \mathrm{ergs} \mathrm{s}^{-1}$. Col. (6): Number of spectroscopically confirmed cluster members within the Chandra field of view more luminous than $M_{R}=-20$ mag. Col. (7): Estimate of the total number of members more luminous than $M_{R}=-20$ mag. Col. (8): AGN fraction if all X-ray sources are AGNs. Col. (9): AGN fraction if all X-ray sources more luminous than $L_{\mathrm{X}}=10^{41} \mathrm{ergs} \mathrm{s}^{-1}$ are AGNs. Col. (10): Same as col. (9), but for X-ray sources more luminous than $L_{\mathrm{X}}=10^{42} \mathrm{ergs} \mathrm{s}^{-1}$. The final two rows present the AGN fraction calculated from the average of the eight clusters and the average of the sum of the eight clusters. See $\S 5.1$ for further details. 


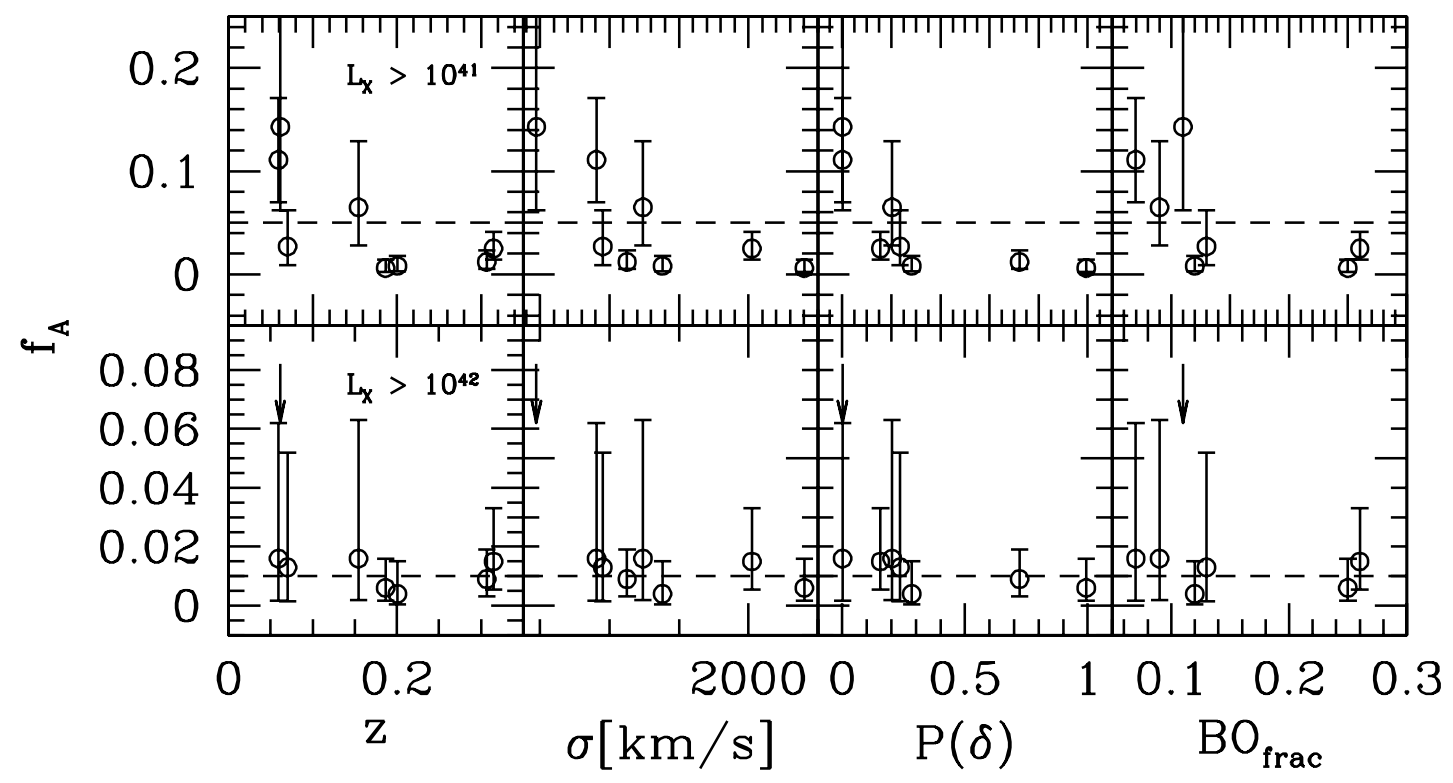

FIG. 10.- AGN fraction as a function of redshift, cluster velocity dispersion, amount of cluster substructure, and estimated fraction of BO galaxies. The AGN fraction

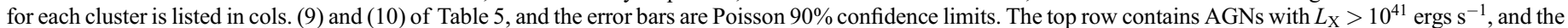

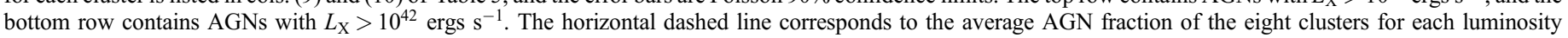
threshold. The cluster properties are provided in Table 1.

relations between AGNs and their host galaxies in the field motivate our choice of these properties. First, a dependence with redshift is expected because of the pronounced decrease in the space density of AGNs from high redshift to the present (e.g., Osmer 2004). In addition, the massive, early-type galaxies in clusters should harbor supermassive black holes that most likely grew by accretion as luminous AGNs at earlier times. Statistical studies of X-ray point-source overdensities toward clusters do show evidence of an increase in the AGN fraction in high-redshift clusters (Dowsett 2005), although those AGNs have not been spectroscopically confirmed to be cluster members. Nevertheless, these arguments suggest that the AGN population in clusters of galaxies should be higher at higher redshift, although they do not necessarily require pronounced evolution over the redshift range of this sample. In particular, most of the AGNs in these clusters are low luminosity $\left(L_{\mathrm{X}} \sim 10^{41}-10^{42} \mathrm{ergs} \mathrm{s}^{-1}\right)$, and current cosmic downsizing models indicate that there is relatively little evolution at these luminosities at low redshift (e.g., Ueda et al. 2003; Hasinger et al. 2005). In fact, the top left panel of Figure 10 actually shows that the AGN fraction increases at lower redshift for the $L_{\mathrm{X}}>10^{41} \mathrm{erg} \mathrm{s}^{-1}$ sample, contrary to these expectations. However, there are substantial correlations between redshift and several other properties of these clusters, and therefore this apparent correlation may be a selection effect. The most important of the potential systematic effects produced by these correlations are due to the two lowest redshift clusters, the merging pair Abell 3125/3128. These two clusters possess the greatest substructure and also have the lowest velocity dispersions. The cluster sample for this survey was simply chosen from available Chandra data in the southern hemisphere to obtain a measurement of the AGN fraction in clusters (see Paper I), and these potential parameter correlations were not foreseen.

Perhaps the most important correlation between two cluster properties in our sample is that between redshift and velocity dispersion. If mergers play an important role in triggering lowluminosity AGNs, we expect that clusters of galaxies with lower velocity dispersions will have a higher AGN fraction because the merger rate will increase. The second column of Figure 10 shows that some of the lower velocity dispersion clusters do have higher AGN fractions, although this trend is largely driven by Abell 3125/3128. Recent evidence for an anticorrelation between AGN fraction and velocity dispersion comes from the work of Popesso \& Biviano (2006), who studied the AGN fraction as a function of velocity dispersion with SDSS spectroscopic data. These authors found that the average AGN fraction increased in environments with lower velocity dispersion.

Finally, the last two columns of Figure 10 show the AGN fraction as a function of cluster substructure $P(\delta)$ and the fraction of BO galaxies in the cluster. The AGN fraction may depend on cluster substructure if cluster-cluster mergers produce a substantial increase in the galaxy interaction rate. In the above discussion of how the merger rate is lower in higher velocity dispersion environments, we neglected the potential role of fast flyby interactions in fueling AGNs. These fast interactions have been studied as a mechanism for driving galaxy evolution in clusters (Moore et al. 1996; Gnedin 2003) and may also provide a mechanism for fueling AGNs (Lake et al. 1998). While it is not clear if the rate of flyby interactions will increase in clusters with substantial substructure, our measurements do indicate that the two merging clusters have the highest AGN fraction. Surprisingly, these clusters also have among the lowest fraction of BO galaxies, and this produces an apparent trend that clusters with fewer star-forming galaxies have a larger AGN fraction. While again this is based on a small sample that consequently does not merit too much interpretation, this is contrary to our expectation that clusters with more star formation and more available cold gas would be those with higher AGN fractions.

\section{SUMMARY}

We have investigated the distribution of AGNs in clusters of galaxies in order to study AGN and galaxy evolution in rich environments. Specific questions that motivated our study include the following: What mechanisms fuel accretion onto supermassive black holes? Is the evolution of AGNs in clusters different from AGN evolution in the field? We have taken a step toward answering these questions with a study of the relative distributions 
of AGNs and inactive galaxies in clusters and how the cluster AGN fraction varies as a function of several cluster properties. Our main results are as follows:

1. The most luminous AGNs $\left(L_{\mathrm{X}}>10^{42} \mathrm{ergs} \mathrm{s}^{-1}\right)$ are more centrally concentrated than cluster galaxies with similar absolute magnitude $M_{R}<-20 \mathrm{mag}$, while less luminous AGNs $\left(L_{\mathrm{X}}>\right.$ $10^{42} \mathrm{ergs} \mathrm{s}^{-1}$ ) have a similar distribution to other cluster members. This greater central concentration of the most luminous members is contrary to our expectation that most AGNs will be triggered in the outskirts of clusters. While this survey does not extend to large enough radius to sample the cluster outskirts, our data do demonstrate that the $L_{\mathrm{X}}>10^{42} \mathrm{erg} \mathrm{s}^{-1}$ AGNs are more centrally concentrated in the cluster than inactive galaxies of the same absolute magnitude and these AGNs are not dominated by galaxies that have recently fallen into the cluster. We would see newly infalling galaxies in projection in the kinematic distribution; however, as the cluster AGNs have a similar kinematic distribution to other cluster galaxies, this suggests that they are not significantly more or less likely to be on a radial orbit than a typical cluster galaxy. Cluster AGNs and inactive cluster galaxies also similarly trace cluster substructure. Future observations that encompass a larger fraction of the virial radial will help to physically separate AGNs in the cores of clusters from any potential AGN population in the cluster outskirts.

2. The AGN fraction in clusters varies between clusters to a greater extent than can be explained by Poisson statistics alone. Specifically, the estimated AGN fraction $f_{A}\left(M_{R}<-20 ; L_{\mathrm{X}}>\right.$ $10^{41}$ ) in cluster galaxies ranges from $14 \%$ to $0.6 \%$ with a mean fraction of $5 \%$ per cluster. While there are some systematic uncertainties due to our estimates of the cluster completeness, the statistical and systematic uncertainties for several clusters remain inconsistent with one another with greater than $90 \%$ confidence. We examined if the variation in AGN fraction from cluster to cluster correlates with any cluster property and found weak evidence that the AGN fraction is higher at lower redshift, in lower velocity dispersion clusters, in clusters with substantial substructure, and in clusters with a smaller fraction of BO galaxies. Unfortunately, our sample of clusters is relatively small and has some substantial correlations between these properties. In particular, the two lowest redshift clusters are also those with the most substructure and the lowest velocity dispersions (Abell 3125 and Abell 3128). Therefore, we cannot conclude which of these properties, or what combination of these properties, sets the AGN fraction in clusters. A larger sample of clusters that more evenly fills this parameter space is needed to determine what parameters set the AGN fraction in clusters of galaxies. These data could then be used to test models of AGN fueling, for example, via the functional form of the scaling between AGN fraction and velocity dispersion.
Together these two results provide evidence that the properties of cluster AGNs, like the properties of cluster galaxies, vary both within clusters and from cluster to cluster. An equally interesting question to answer is if the AGN fraction varies substantially between clusters and lower density groups and the field. While evidence from spectroscopic surveys unequivocally answers yes to this question and demonstrates that AGNs are less commonly found in clusters than the field, radio and now our X-ray observations indicate that the dependence of AGN fraction on environment may be much less stark when the AGNs are selected at these other wavelengths. While differences in the typical spectral properties of AGNs as a function of environment do not affect the present comparison between and within clusters, they will complicate comparisons of the AGN fraction between the field, groups, and clusters of galaxies. A possible resolution is that the AGN fraction may be a function of morphological type and not just absolute magnitude. This is known to be the case for spectroscopically classified AGNs (Ho et al. 1997) in the sense that early-type galaxies have a higher incidence of AGNs. As Lehmer et al. (2007) measured a comparable X-ray-selected AGN fraction in morphologically classified early-type galaxies, and these dominate in clusters but not groups, future measurements of the AGN fraction may need to consider morphological type, in addition to host luminosity and AGN luminosity. This may reflect the fact that morphological type sets the spheroid mass and consequently the black hole mass for a galaxy of a given total luminosity.

We would like to thank R. Gilmour (née Dowsett) for helpful discussions, the referee for helpful suggestions, and B. Lehmer and M. Sun for providing us with more information about their measurements of the AGN fraction. Support for this work was provided by the National Aeronautics and Space Administration through Chandra Awards 04700793 and 05700786 issued by the Chandra X-Ray Center, which is operated by the Smithsonian Astrophysical Observatory for and on behalf of the National Aeronautics Space Administration under contract NAS8-03060. We greatly appreciate the excellent staffs of the Las Campanas Observatory and the Magellan Project Telescopes for their assistance with these observations. This paper includes data gathered with the $6.5 \mathrm{~m}$ Magellan Telescopes located at Las Campanas Observatory, Chile. This research has made use of the NASA/ IPAC Extragalactic Database (NED), which is operated by the Jet Propulsion Laboratory, California Institute of Technology, under contract with the National Aeronautics and Space Administration.

Facilities: du Pont (Tek No. 5 imaging CCD, WFCCD), Magellan:Clay (LDSS2 imaging spectrograph)
Abell, G. O., Corwin, H. G., Jr., \& Olowin, R. P. 1989, ApJS, 70, 1

Barnes, J. E., \& Hernquist, L. 1992, ARA\&A, 30, 705

Beers, T. C., Flynn, K., \& Gebhardt, K. 1990, AJ, 100, 32

Best, P. N., Kauffmann, G., Heckman, T. M., Brinchmann, J., Charlot, S., Ivezić, Ž., \& White, S. D. M. 2005, MNRAS, 362, 25

Bîrzan, L., Rafferty, D. A., McNamara, B. R., Wise, M. W., \& Nulsen, P. E. J. 2004, ApJ, 607, 800

Burns, J. O. 1990, AJ, 99, 14

Butcher, H., \& Oemler, A. 1978, ApJ, 219, 18

Cappi, M., et al. 2001, ApJ, 548, 624

Carlberg, R. G., Yee, H. K. C., \& Ellingson, E. 1997, ApJ, 478, 462

Coble, K., Carlstrom, J. E., Bonamente, M., Dawson, K., Holzapfel, W., Joy, M., LaRoque, S., \& Reese, E. D. 2006, AJ, submitted (astro-ph/0608274)

\section{REFERENCES}

Couch, W. J., \& Sharples, R. M. 1987, MNRAS, 229, 423

Czoske, O. 2004, in IAU Colloq. 195, Outskirts of Galaxy Clusters: Intense Life in the Suburbs, ed. A. Diaferio (Cambridge: Cambridge Univ. Press), 183

de Robertis, M. M., Yee, H. K. C., \& Hayhoe, K. 1998, ApJ, 496, 93

Dowsett, R. 2005, Ph.D. thesis

Dressler, A. 1980, ApJ, 236, 351

Dressler, A., \& Shectman, S. A. 1988, AJ, 95, 284

Dressler, A., Smail, I., Poggianti, B. M., Butcher, H., Couch, W. J., Ellis, R. S., \& Oemler, A. J. 1999, ApJS, 122, 51

Dressler, A., Thompson, I. B., \& Shectman, S. A. 1985, ApJ, 288, 481

Fabian, A. C., et al. 2000, MNRAS, 318, L65

Fabricant, D. G., McClintock, J. E., \& Bautz, M. W. 1991, ApJ, 381, 33 
Fisher, D., Fabricant, D., Franx, M., \& van Dokkum, P. 1998, ApJ, 498, 195 Giovanelli, R., \& Haynes, M. P. 1985, ApJ, 292, 404

Gisler, G. R. 1978, MNRAS, 183, 633

Gnedin, O. Y. 2003, ApJ, 589, 752

Hasinger, G., Miyaji, T., \& Schmidt, M. 2005, A\&A, 441, 417

Ho, L. C., Filippenko, A. V., \& Sargent, W. L. W. 1997, ApJ, 487, 568

Hopkins, P. F., Hernquist, L., Martini, P., Cox, T. J., Robertson, B., Di Matteo, T., \& Springel, V. 2005, ApJ, 625, L71

Kim, D.-W., \& Fabbiano, G. 2004, ApJ, 611, 846

Lake, G., Katz, N., \& Moore, B. 1998, ApJ, 495, 152

Lehmer, B. D., et al. 2007, ApJ, 657, 681

Liang, H., Lémonon, L., Valtchanov, I., Pierre, M., \& Soucail, G. 2000, A\&A, 363,440

Lin, Y.-T., \& Mohr, J. J. 2007, ApJS, 170, 71

Martini, P. 2004, in IAU Symp. 222, The Interplay among Black Holes, Stars and ISM in Galactic Nuclei, ed. T. Storchi-Bergmann, L. C. Ho, \& H. R. Schmitt (Cambridge: Cambridge Univ. Press), 235

Martini, P., Kelson, D. D., Kim, E., Mulchaey, J. S., \& Athey, A. A. 2006, ApJ, 644, 116 (Paper I)

Martini, P., Kelson, D. D., Mulchaey, J. S., \& Trager, S. C. 2002, ApJ, 576, L109

McNamara, B. R., et al. 2000, ApJ, 534, L135

Molnar, S. M., Hughes, J. P., Donahue, M., \& Joy, M. 2002, ApJ, 573, L91

Moore, B., Katz, N., Lake, G., Dressler, A., \& Oemler, A., Jr. 1996, Nature, 379,613
Morrison, G. E., Owen, F. N., Ledlow, M. J., Keel, W. C., Hill, J. M., Voges, W., \& Herter, T. 2003, ApJS, 146, 267

Nusser, A., Silk, J., \& Babul, A. 2006, MNRAS, 373, 739

Osmer, P. S. 2004, in Coevolution of Black Holes and Galaxies, ed. L. C. Ho (Cambridge: Cambridge Univ. Press), 324

Osterbrock, D. E. 1960, ApJ, 132, 325

O'Sullivan, E., Ponman, T. J., \& Collins, R. S. 2003, MNRAS, 340, 1375

Popesso, P., \& Biviano, A. 2006, A\&A, 460, L23

Rose, J. A., Gaba, A. E., Christiansen, W. A., Davis, D. S., Caldwell, N., Hunstead, R. W., \& Johnston-Hollitt, M. 2002, AJ, 123, 1216

Ruderman, J. T., \& Ebeling, H. 2005, ApJ, 623, L81

Schmitt, H. R. 2001, AJ, 122, 2243

Shen, Y., Mulchaey, J. S., Raychaudhury, S., Rasmussen, J., \& Ponman, T. J. 2007, ApJ, 654, L115

Sun, M., Jones, C., Forman, W., Vikhlinin, A., Donahue, M., \& Voit, M. 2007, ApJ, 657, 197

Sun, M., \& Murray, S. S. 2002, ApJ, 577, 139

Sun, M., Vikhlinin, A., Forman, W., Jones, C., \& Murray, S. S. 2005, ApJ, 619, 169

Teague, P. F., Carter, D., \& Gray, P. M. 1990, ApJS, 72, 715

Treu, T., Ellis, R. S., Kneib, J.-P., Dressler, A., Smail, I., Czoske, O., Oemler, A., \& Natarajan, P. 2003, ApJ, 591, 53

Ueda, Y., Akiyama, M., Ohta, K., \& Miyaji, T. 2003, ApJ, 598, 886 